\title{
Efficient Numerical Solution of Parabolic Optimization Problems by Finite Element Methods
}

\author{
Roland Becker ${ }^{\dagger}$, Dominik Meidner ${ }^{\ddagger}$, and Boris Vexler $₫$
}

\begin{abstract}
We present an approach for efficient numerical solution of optimization problems governed by parabolic partial differential equations. The main ingredients are: space-time finite element discretization, second order optimization algorithms and storage reduction techniques. We discuss the combination of these components for the solution of large scale optimization problems.
\end{abstract}

Keywords: optimal control, parabolic equations, finite elements, storage reduction

AMS Subject Classification:

\section{Introduction}

In this paper, we discuss efficient numerical methods for solving optimization problems governed by parabolic partial differential equations. The optimization problems are formulated in a general setting including optimal control as well as parameter identification problems. Both, time and space discretization are based on the finite element method. This allows a natural translation of the optimality conditions from the continuous to the discrete level. For this type of discretizations, we present a systematic approach for precise computation of the derivatives required in optimization algorithms. The evaluation of these derivatives is based on the solutions of appropriate adjoint (dual) and sensitivity (tangent) equations.

The solution of the underlying state equation is typically required in the whole time interval for the computation of these additional solutions. If all data are stored, the storage grows linearly with respect to the number of time

\footnotetext{
†Laboratoire de Mathématiques Appliquées, Université de Pau et des Pays de l'Adour BP 1155, 64013 PAU Cedex, France

${ }^{\ddagger}$ Institut für Angewandte Mathematik, Ruprecht-Karls-Universität Heidelberg, INF 294, 69120 Heidelberg, Germany. This work has been partially supported by the German Research Foundation (DFG) through the Internationales Graduiertenkolleg 710 'Complex Processes: Modeling, Simulation, and Optimization'

I Johann Radon Institute for Computational and Applied Mathematics (RICAM), Austrian Academy of Sciences, Altenberger Straße 69, 4040 Linz, Austria
} 
intervals in the time-discretization. This makes the optimization procedure prohibitive for fine discretizations. We suggest an algorithm, which allows to reduce the required storage. We analyze the complexity of this algorithm and prove that the required storage grows only logarithmic with respect to the number of time intervals. Such results are well-known for gradient evaluations in the context of automatic differentiation, see Griewank [12,13] and Griewank and Walther [14]. However, to the authors' knowledge, the analysis of the required numerical effort for the whole optimization algorithm is new. The presented approach is an extension of windowing strategies introduced in Berggren, Glowinski, and Lions [5].

The main contribution of this paper is the combination of the exact computation of the derivatives based on the space-time finite element discretization with the storage reduction techniques.

In this paper, we consider optimization problems under constraints of (nonlinear) parabolic differential equations

$$
\begin{aligned}
\partial_{t} u+A(q, u) & =f \\
u(0) & =u_{0}(q) .
\end{aligned}
$$

Here, the state variable is denoted by $u$ and the control variable by $q$. Both, the differential operator $A$ and the initial condition $u_{0}$ may depend on $q$. This allows a simultaneous treatment of both, optimal control and parameter identification problems. For optimal control problems, the operator $A$ is typically given by

$$
A(q, u)=\bar{A}(u)+B(q),
$$

with a (nonlinear) operator $\bar{A}$ and (usually linear) control operator $B$. In parameter identification problems, the variable $q$ denotes the unknown parameters to be determined and may enter the operator $A$ in a nonlinear way. The case of initial control is included via the $q$-dependent initial condition $u_{0}(q)$.

The target of the optimization is to minimize a given cost functional $J(q, u)$ subject to the state equation (1).

For covering additional constraints on the control variable, one may seek $q$ in an admissible set describing, e.g., box constraints on $q$. For clarity of presentation, we consider here the case of no additional control contraints. However, the algorithms discussed in the sequel, can be used as an interior loop within a primal-dual active set strategy, see, e.g., Bergounioux, Ito, and Kunisch [6] and Kunisch and Rösch [16].

The paper is organized as follows: In the next section we describe an abstract optimization problem, with a parabolic state equation written in a weak form and discuss optimality conditions. Then, the problem is reformulated as an 
unconstrained (reduced) optimization problem and the expressions for the required derivatives are provided. After that, we describe Newton-type methods for solution of the problem on the continuous level. In Section 3, we discuss the space and time discretizations. The space discretization is done by conforming finite elements and for the time discretization we use two approaches: discontinuous Galerkin (dG) and continuous Galerkin (cG) methods, see, e.g., Eriksson, Johnson, and Thomée [9]. For both, we provide techniques for precise evaluation of the derivatives in the corresponding discrete problems. This allows for simple translation of the optimization algorithm described in Section 2 from the continuous to the discrete level. Section 4 is devoted to the storage reduction techniques. Here, we present and analyze an algorithm, which we call Multi-Level Windowing, allowing for drastically reduction of the required storage by the computation of adjoint solutions. This algorithm is then specified for the computation of the derivatives required in the optimization loop. In the last section we present numerical results illustrating our approach.

\section{Optimization}

The optimization problems considered in this paper, are formulated in the following abstract setting: Let $Q$ be a Hilbert space for the controls (parameters) with scalar product $(\cdot, \cdot)_{Q}$. Moreover, let $V$ and $H$ be Hilbert spaces, which build together with the dual space $V^{*}$ a Gelfand triple: $V \hookrightarrow H \hookrightarrow V^{*}$. The duality pairing between the Hilbert space $V$ and its dual $V^{*}$ is denoted by $\langle\cdot, \cdot\rangle_{V^{*} \times V}$ and the scalar product in $H$ by $(\cdot, \cdot)_{H}$.

Remark 2.1 By the definition of the Gelfand triple, the space $H$ is dense in $V^{*}$. Therefore, every functional $v^{*} \in V^{*}$ can be uniformly approximated by scalar products in $H$. That is, we can regard the continuous continuation of $(\cdot, \cdot)_{H}$ onto $V \times V^{*}$ as new representation formula for the functionals in $V^{*}$.

Let, moreover, $I=(0, T)$ be a time interval and the space $X$ be defined as

$$
X=\left\{v \mid v \in L^{2}(I, V) \text { and } \partial_{t} v \in L^{2}\left(I, V^{*}\right)\right\} .
$$

It is well known, that the space $X$ is continuously embedded in $C(\bar{I}, H)$, see, e.g., Dautray and Lions [8].

After these preliminaries, we pose the state equation in a weak form using the form $a(\cdot, \cdot)(\cdot)$ defined on $Q \times V \times V$, which is assumed to be twice continuously differentiable and linear in the third argument. The state variable $u \in X$ is 
determined by

$$
\begin{aligned}
\int_{0}^{T}\left\{\left(\partial_{t} u, \varphi\right)_{H}+a(q, u)(\varphi)\right\} d t & =\int_{0}^{T}(f, \varphi)_{H} d t \quad \forall \varphi \in X, \\
u(0) & =u_{0}(q),
\end{aligned}
$$

where $f \in L^{2}\left(0, T ; V^{*}\right)$ represents the right hand side of the state equation and $u_{0}: Q \rightarrow H$ denotes a twice continuously differentiable mapping describing parameter-dependent initial conditions. Note, that the scalar products $\left(\partial_{t} u, \varphi\right)_{H}$ and $(f, \varphi)_{H}$ have to be understood according to Remark 2.1. For brevity of notation, we omit the arguments $t$ and $x$ of time-dependent functions whenever possible.

The cost functional $J: Q \times X \rightarrow \mathbb{R}$ is defined using two twice continuously differentiable functionals $I: V \rightarrow \mathbb{R}$ and $K: H \rightarrow \mathbb{R}$ by:

$$
J(q, u)=\int_{0}^{T} I(u) d t+K(u(T))+\frac{\alpha}{2}\|q-\bar{q}\|_{Q}^{2},
$$

where the regularization (or cost) term involving $\alpha \geq 0$ and a reference parameter $\bar{q} \in Q$ is added.

The corresponding optimization problem is formulated as follows:

$$
\text { Minimize } J(q, u) \text { subject to }(3),(q, u) \in Q \times X \text {. }
$$

The question of existence and uniqueness of solutions to such optimization problems is discussed in, e.g., Lions [17], Fursikov [11], and Litvinov [18]. Throughout the paper, we assume problem (5) to admit a (locally) unique solution.

Furthermore, under a regularity assumption on $a_{u}^{\prime}(q, u)$ at the solution of (5), the implicit function theorem ensures the existence of an open subset $Q_{0} \subset Q$ containing the solution of the optimization problem under consideration, and of a twice continuously differentiable solution operator $S: Q_{0} \rightarrow X$ of the state equation (3). Thus, for all $q \in Q_{0}$ we have

$$
\begin{aligned}
\int_{0}^{T}\left\{\left(\partial_{t} S(q), \varphi\right)_{H}+a(q, S(q))(\varphi)\right\} d t & =\int_{0}^{T}(f, \varphi)_{H} d t \quad \forall \varphi \in X, \\
S(q)(0) & =u_{0}(q) .
\end{aligned}
$$

Using this solution operator we introduce the reduced cost functional $j: Q_{0} \rightarrow \mathbb{R}$, defined by $j(q)=J(q, S(q))$. This definition allows to reformulate 
problem (5) as an unconstrained optimization problem:

$$
\text { Minimize } j(q), \quad q \in Q_{0} \text {. }
$$

If $q$ is an optimal solution of the unconstrained problem above, the first and second order necessary optimality condition are fulfilled:

$$
\begin{aligned}
j^{\prime}(q)(\tau q) & =0, & & \forall \tau q \in Q, \\
j^{\prime \prime}(q)(\tau q, \tau q) & \geq 0, & & \forall \tau q \in Q .
\end{aligned}
$$

For the unconstrained optimization problem (7), a second order sufficient optimality condition is given by the positive definiteness of the second derivatives $j^{\prime \prime}(q)$.

To express the first and second derivatives of the reduced cost functional $j$, we introduce the Lagrangian $\mathcal{L}: Q \times X \times X \times H \rightarrow \mathbb{R}$, defined as

$$
\begin{aligned}
\mathcal{L}(q, u, z, \tilde{z})= & J(q, u) \\
& +\int_{0}^{T}\left\{\left(f-\partial_{t} u, z\right)_{H}-a(q, u)(z)\right\} d t-\left(u(0)-u_{0}(q), \tilde{z}\right)_{H} .
\end{aligned}
$$

With the help of the Lagrangian, we now present three auxiliary equations, which we will use in the sequel to give expressions of the derivatives of the reduced functional. Each equation will thereby be given in two formulations, first in terms of the Lagrangian and then using the concrete form of the optimization problem under consideration.

- Dual Equation: For given $q \in Q_{0}$ and $u=S(q) \in X$, find $(z, \tilde{z}) \in X \times H$ such that

$$
\mathcal{L}_{u}^{\prime}(q, u, z, \tilde{z})(\varphi)=0, \quad \forall \varphi \in X .
$$

- Tangent Equation: For given $q \in Q_{0}, u=S(q) \in X$, and a given direction $\delta q \in Q$, find $\delta u \in X$ such that

$$
\begin{aligned}
\mathcal{L}_{q z}^{\prime \prime}(q, u, z, \tilde{z})(\delta q, \varphi) & +\mathcal{L}_{u z}^{\prime \prime}(q, u, z, \tilde{z})(\delta u, \varphi)+\mathcal{L}_{q \tilde{z}}^{\prime \prime}(q, u, z, \tilde{z})(\delta q, \psi) \\
& +\mathcal{L}_{u \tilde{z}}^{\prime \prime}(q, u, z, \tilde{z})(\delta u, \psi)=0, \quad \forall(\varphi, \psi) \in X \times H .
\end{aligned}
$$

- Dual for Hessian Equation: For given $q \in Q_{0}, u=S(q) \in X,(z, \tilde{z}) \in X \times H$ the corresponding solution of the dual equation (9), and $\delta u \in X$ the solution of the tangent equation (10) for the given direction $\delta q$, find $(\delta z, \delta \tilde{z}) \in X \times H$ 
such that

$$
\begin{aligned}
\mathcal{L}_{q u}^{\prime \prime}(q, u, z, \tilde{z})(\delta q, \varphi)+\mathcal{L}_{u u}^{\prime \prime}(q, u, z, \tilde{z})(\delta u, \varphi) \\
\quad+\mathcal{L}_{z u}^{\prime \prime}(q, u, z, \tilde{z})(\delta z, \varphi)+\mathcal{L}_{\tilde{z} u}^{\prime \prime}(q, u, z, \tilde{z})(\delta \tilde{z}, \varphi)=0, \quad \forall \varphi \in X
\end{aligned}
$$

Equivalently, we may rewrite these equations more detailed in the following way:

- Dual Equation: For given $q \in Q_{0}$ and $u=S(q) \in X$, find $(z, \tilde{z}) \in X \times H$ such that

$$
\begin{aligned}
\int_{0}^{T}\left\{-\left(\varphi, \partial_{t} z\right)_{H}+a_{u}^{\prime}(q, u)(\varphi, z)\right\} d t & =\int_{0}^{T} I^{\prime}(u)(\varphi) d t, \quad \forall \varphi \in X, \\
z(T) & =K^{\prime}(u(T)), \\
\tilde{z} & =z(0) .
\end{aligned}
$$

- Tangent Equation: For $q \in Q_{0}, u=S(q) \in X$, and a given direction $\delta q \in Q$, find $\delta u \in X$ such that

$$
\begin{aligned}
\int_{0}^{T}\left\{\left(\partial_{t} \delta u, \varphi\right)_{H}+a_{u}^{\prime}(q, u)(\delta u, \varphi)\right\} d t & =-\int_{0}^{T} a_{q}^{\prime}(q, u)(\delta q, \varphi) d t, \quad \forall \varphi \in X, \\
\delta u(0) & =u_{0}^{\prime}(q)(\delta q) .
\end{aligned}
$$

- Dual for Hessian Equation: For given $q \in Q_{0}, u=S(q) \in X,(z, \tilde{z}) \in X \times H$ the corresponding solution of the dual equation (12), and $\delta u \in X$ the solution of the tangent equation (13) for the given direction $\delta q$, find $(\delta z, \delta \tilde{z}) \in X \times H$ such that

$$
\begin{gathered}
\int_{0}^{T}\left\{-\left(\varphi, \partial_{t} \delta z\right)_{H}+a_{u}^{\prime}(q, u)(\varphi, \delta z)\right\} d t=\int_{0}^{T} I^{\prime \prime}(u)(\delta u, \varphi) d t \\
-\int_{0}^{T}\left\{a_{u u}^{\prime \prime}(q, u)(\delta u, \varphi, z)+a_{q u}^{\prime \prime}(q, u)(\delta q, \varphi, z)\right\} d t, \quad \forall \varphi \in X, \\
\delta z(T)=K^{\prime \prime}(u(T))(\delta u(T)), \\
\delta \tilde{z}=\delta z(0) .
\end{gathered}
$$

To get the representation (13) of the tangent equation from (10), we only need to calculate the derivatives of the Lagrangian (8). The derivation of the representations (12) and (14) for the dual and the dual for Hessian equation requires more care. Here, we integrate by parts and separate the arising boundary terms by appropriate variation of the test functions. 
In virtue of the dual equation defined above, we can now state an expression for the first derivatives of the reduced functional:

TheOREM 2.1 Let for given $q \in Q_{0}$ :

(i) $u=S(q) \in X$ be a solution of the state equation (3).

(ii) $(z, \tilde{z}) \in X \times H$ fulfill the dual equation (12).

Then there holds

$$
j^{\prime}(q)(\tau q)=\mathcal{L}_{q}^{\prime}(q, u, z, \tilde{z})(\tau q), \quad \forall \tau q \in Q
$$

which we may expand as

$j^{\prime}(q)(\tau q)=\alpha(q-\bar{q}, \tau q)_{Q}-\int_{0}^{T} a_{q}^{\prime}(q, u)(\tau q, z) d t+\left(u_{0}^{\prime}(q)(\tau q), \tilde{z}\right)_{H}, \quad \forall \tau q \in Q$.

Proof Since condition (i) ensures that $u$ is the solution of the state equation (3), and due to both, the definition (6) of the solution operator $S$ and the definition (8) of the Lagrangian, we obtain:

$$
j(q)=\mathcal{L}(q, u, z, \tilde{z}) .
$$

By taking (total) derivative of (16) with respect to $q$ in direction $\tau q$, we get

$$
\begin{aligned}
j^{\prime}(q)(\tau q)= & \mathcal{L}_{q}^{\prime}(q, u, z, \tilde{z})(\tau q)+\mathcal{L}_{u}^{\prime}(q, u, z, \tilde{z})(\tau u) \\
& +\mathcal{L}_{z}^{\prime}(q, u, z, \tilde{z})(\tau z)+\mathcal{L}_{\tilde{z}}^{\prime}(q, u, z, \tilde{z})(\tau \tilde{z}),
\end{aligned}
$$

where $\tau u=S^{\prime}(q)(\tau q)$, and $\tau z \in X$ as well as $\tau \tilde{z} \in H$ are the derivatives of $z$ or respectively $\tilde{z}$ with respect to $q$ in direction $\tau q$. Noting the equivalence of condition (i) with

$$
\mathcal{L}_{z}^{\prime}(q, u, z, \tilde{z})(\varphi)+\mathcal{L}_{\tilde{z}}^{\prime}(q, u, z, \tilde{z})(\psi)=0, \quad \forall(\varphi, \psi) \in X \times H,
$$

and calculating the derivative of the Lagrangian (8) completes the proof.

To use Newton's method for solving the considered optimization problems, we have to compute the second derivatives of the reduced functional. The following theorem presents two alternatives for doing that. These two versions lead to two different optimization loops, which are presented in the sequel.

TheOREm 2.2 Let for given $q \in Q_{0}$ the conditions of Theorem 2.1 be fulfilled.

(a) Moreover, let for given $\delta q \in Q$ : 
(i) $\delta u \in X$ fulfill the tangent equation (13).

(ii) $(\delta z, \delta \tilde{z}) \in X \times H$ fulfill the dual for Hessian equation (14).

Then there holds

$$
\begin{aligned}
j^{\prime \prime}(q)(\delta q, \tau q) & =\mathcal{L}_{q q}^{\prime \prime}(q, u, z, \tilde{z})(\delta q, \tau q)+\mathcal{L}_{u q}^{\prime \prime}(q, u, z, \tilde{z})(\delta u, \tau q) \\
+ & \mathcal{L}_{z q}^{\prime \prime}(q, u, z, \tilde{z})(\delta z, \tau q)+\mathcal{L}_{\tilde{z} q}^{\prime \prime}(q, u, z, \tilde{z})(\delta \tilde{z}, \tau q), \quad \forall \tau q \in Q
\end{aligned}
$$

which we may equivalently express as

$$
\begin{aligned}
& j^{\prime \prime}(q)(\delta q, \tau q)=\alpha(\delta q, \tau q)_{Q} \\
& -\int_{0}^{T}\left\{a_{q q}^{\prime \prime}(q, u)(\delta q, \tau q, z)+a_{u q}^{\prime \prime}(q, u)(\delta u, \tau q, z)+a_{q}^{\prime}(q, u)(\tau q, \delta z)\right\} d t \\
& \quad+\left(u_{0}^{\prime}(q)(\tau q), \delta \tilde{z}\right)_{H}+\left(u_{0}^{\prime \prime}(q)(\delta q, \tau q), \tilde{z}\right)_{H}, \quad \forall \tau q \in Q . \quad
\end{aligned}
$$

(b) Moreover, let for given $\delta q, \tau q \in Q$ :

(i) $\delta u \in X$ fulfill the tangent equation (13) for the given direction $\delta q$.

(ii) $\tau u \in X$ fulfill the tangent equation (13) for the given direction $\tau q$.

Then there holds

$$
\begin{aligned}
j^{\prime \prime}(q)(\delta q, \tau q)=\mathcal{L}_{q q}^{\prime \prime}(q, u, z, \tilde{z})(\delta q, \tau q)+\mathcal{L}_{u q}^{\prime \prime}(q, u, z, \tilde{z})(\delta u, \tau q) \\
+\mathcal{L}_{q u}^{\prime \prime}(q, u, z, \tilde{z})(\delta q, \tau u)+\mathcal{L}_{u u}^{\prime \prime}(q, u, z, \tilde{z})(\delta u, \tau u)
\end{aligned}
$$

which we may equivalently express as

$$
\begin{aligned}
& j^{\prime \prime}(q)(\delta q, \tau q)=\alpha(\delta q, \tau q)_{Q}+\int_{0}^{T} I_{u u}^{\prime \prime}(u)(\delta u, \tau u) d t \\
& -\int_{0}^{T}\left\{a_{q q}^{\prime \prime}(q, u)(\delta q, \tau q, z)+a_{u q}^{\prime \prime}(q, u)(\delta u, \tau q, z)+a_{q u}^{\prime \prime}(q, u)(\delta q, \tau u, z)\right. \\
& \left.\quad+a_{u u}^{\prime \prime}(q, u)(\delta u, \tau u, z)\right\} d t+K_{u u}^{\prime \prime}(u)(\delta u, \tau u) .
\end{aligned}
$$

Proof Due to condition (i) of Theorem 2.1, we obtain as before

$$
\begin{aligned}
j^{\prime}(q)(\delta q)= & \mathcal{L}_{q}^{\prime}(q, u, z, \tilde{z})(\delta q)+\mathcal{L}_{u}^{\prime}(q, u, z, \tilde{z})(\delta u) \\
& +\mathcal{L}_{z}^{\prime}(q, u, z, \tilde{z})(\delta z)+\mathcal{L}_{\tilde{z}}^{\prime}(q, u, z, \tilde{z})(\delta \tilde{z}),
\end{aligned}
$$


and taking (total) derivatives with respect to $q$ in direction $\tau q$ yields

$$
\begin{aligned}
j^{\prime \prime}(q)(\delta q, \tau q)= & \\
& \mathcal{L}_{q q}^{\prime}()(\delta q, \tau q)+\mathcal{L}_{q u}^{\prime}()(\delta q, \tau u)+\mathcal{L}_{q z}^{\prime}()(\delta q, \tau z)+\mathcal{L}_{q \tilde{z}}^{\prime}()(\delta q, \tau \tilde{z}) \\
+ & \mathcal{L}_{u q}^{\prime}()(\delta u, \tau q)+\mathcal{L}_{u u}^{\prime}()(\delta u, \tau u)+\mathcal{L}_{u z}^{\prime}()(\delta u, \tau z)+\mathcal{L}_{u \tilde{z}}^{\prime}()(\delta u, \tau \tilde{z}) \\
+ & \mathcal{L}_{z q}^{\prime}()(\delta z, \tau q)+\mathcal{L}_{z u}^{\prime}()(\delta z, \tau u) \\
+ & \mathcal{L}_{\tilde{z} q}^{\prime}()(\delta \tilde{z}, \tau q)+\mathcal{L}_{\tilde{z} u}^{\prime}()(\delta \tilde{z}, \tau u) \\
& +\mathcal{L}_{u}^{\prime}()\left(\delta^{2} u\right)+\mathcal{L}_{z}^{\prime}()\left(\delta^{2} z\right)+\mathcal{L}_{\tilde{z}}^{\prime}()\left(\delta^{2} \tilde{z}\right) .
\end{aligned}
$$

(For abbreviation we have omitted the content of the first parenthesis of the Lagrangian.) In addition to the notations in the proof of Theorem 2.1, we have defined $\delta^{2} u=S^{\prime \prime}(q)(\delta q, \tau q)$, and $\delta^{2} z \in X$ as well as $\delta^{2} \tilde{z} \in H$ as the second derivatives of $z$ or respectively $\tilde{z}$ in the directions $\delta q$ and $\tau q$.

We complete the proof applying the stated conditions to this expression.

In the sequel, we present two variants of the Newton based optimization loop on the continuous level. The difference between these variants consists in the way of computing the update. Newton-type methods are used for solving optimization problem governed by time-dependent partial differential equations, see, e.g., Hinze and Kunisch [15] and Tröltzsch [20].

From here on, we consider finite dimensional control space $Q$ with a basis:

$$
\left\{\tau q_{i} \mid i=1, \ldots, \operatorname{dim} Q\right\} .
$$

Both, Algorithm 2.1 and Algorithm 2.3, describe an usual Newton-type method for the unconstrained optimization problem (7), which requires the solution of the following linear system in each iteration:

$$
\nabla^{2} j(q) \delta q=-\nabla j(q)
$$

where the gradient $\nabla j(q)$ and the Hessian $\nabla^{2} j(q)$ are defined as usual by the identifications:

$$
\begin{gathered}
(\nabla j(q), \tau q)_{Q}=j^{\prime}(q)(\tau q), \quad \forall \tau q \in Q, \\
\left(\tau q, \nabla^{2} j(q) \delta q\right)_{Q}=j^{\prime \prime}(q)(\delta q, \tau q), \quad \forall \delta q, \tau q \in Q .
\end{gathered}
$$

In both algorithms, the required gradient $\nabla j(q)$ is computed using representation (15) from Theorem 2.1. However, the algorithms differ in the way how 
they solve the linear system (20) to obtain a correction $\delta q$ for the current control $q$. Algorithm 2.1 treats the computation of this system using the conjugate gradients method. It basically necessitates products of the Hessian with given vectors and does not need the entire Hessian.

Algorithm 2.1 Optimization Loop without building up the Hessian:

1: Choose initial $q^{0} \in Q_{0}$ and set $n=0$.

2: repeat

3: $\quad$ Compute $u^{n} \in X$, i.e. solve the state equation (3).

4: $\quad$ Compute $\left(z^{n}, \tilde{z}^{n}\right) \in X \times H$, i.e. solve the dual equation (12).

5: $\quad$ Build up the gradient $\nabla j\left(q^{n}\right)$. To compute its $i$-th component $\left(\nabla j\left(q^{n}\right)\right)_{i}$, evaluate the right hand side of representation (15) for $\tau q=\tau q_{i}$.

6: $\quad$ Solve

$$
\nabla^{2} j\left(q^{n}\right) \delta q=-\nabla j\left(q^{n}\right)
$$

by use of the method of conjugate gradients.(For the computation of the required matrix-vector products, apply the procedure described in Algorithm 2.2.)

7: $\quad$ Set $q^{n+1}=q^{n}+\delta q$.

8: $\quad$ Increment $n$.

9: until $\left\|\nabla j\left(q^{n}\right)\right\|<$ TOL

The computation of the required matrix-vector products can be done with the representation given in Theorem 2.2(a) and is described in Algorithm 2.2. We note that in order to obtain the product of the Hessian with a given vector, we have to solve one tangent equation and one dual for Hessian equation. This has to be done in each step of the method of conjugate gradients.

Algorithm 2.2 Computation of the matrix-vector product $\nabla^{2} j\left(q^{n}\right) \delta q$ :

Require: $u^{n}, z^{n}$, and $\tilde{z}^{n}$ are already computed for the given $q^{n}$

1: Compute $\delta u^{n} \in X$, i.e. solve the tangent equation (13).

2: Compute $\left(\delta z^{n}, \delta \tilde{z}^{n}\right) \in X \times H$, i.e. solve the dual for Hessian equation (14).

3: Build up the product $\nabla^{2} j\left(q^{n}\right) \delta q$. To compute its $i$-th component $\left(\nabla^{2} j\left(q^{n}\right) \delta q\right)_{i}$, evaluate the right hand side of representation (17) for $\tau q=\tau q_{i}$.

In contrast to Algorithm 2.1, Algorithm 2.3 builds up the whole Hessian. Consequently we may use every linear solver to the linear system (20). To compute the Hessian, we use the representation of the second derivatives of the reduced functional given in Theorem 2.2(b). Thus, in each Newton step we have to solve the tangent equation for each basis vector in (19).

Algorithm 2.3 Optimization Loop with building up the Hessian: 
1: Choose initial $q^{0} \in Q_{0}$ and set $n=0$.

2: repeat

Compute $u^{n} \in X$, i.e. solve the state equation (3).

Compute $\left\{\tau u_{i}^{n} \mid i=1, \ldots, \operatorname{dim} Q\right\} \subset X$ for the chosen basis of $Q$, i.e. solve the tangent equation (13) for each of the basis vectors $\tau q_{i}$ in (19).

5: $\quad$ Compute $z^{n} \in X$, i.e. solve the dual equation (12).

6: $\quad$ Build up the gradient $\nabla j\left(q^{n}\right)$. To compute its $i$-th component $\left(\nabla j\left(q^{n}\right)\right)_{i}$, evaluate the right hand side of representation (15) for $\tau q=\tau q_{i}$.

7: $\quad$ Build up the Hessian $\nabla^{2} j\left(q^{n}\right)$. To compute its $i j$-th entry $\left(\nabla^{2} j\left(q^{n}\right)\right)_{i j}$, evaluate the right hand side of representation (18) for $\delta q=\tau q_{j} \tau q=\tau q_{i}$, $\delta u=\tau u_{j}$, and $\tau u=\tau u_{i}$.

8: Compute $\delta q$ as the solution of

$$
\nabla^{2} j\left(q^{n}\right) \delta q=-\nabla j\left(q^{n}\right)
$$

by use of an arbitrary linear solver.

9: $\quad$ Set $q^{n+1}=q^{n}+\delta q$.

10: $\quad$ Increment $n$.

11: until $\left\|\nabla j\left(q^{n}\right)\right\|<\mathrm{TOL}$

We now compare the efficiency of the two presented algorithms. For one step of Newton's method, Algorithm 2.1 requires the solution of two linear problems (tangent equation and dual for Hessian equation) for each step of the CG-iteration, whereas for Algorithm 2.3 it is necessary to solve $\operatorname{dim} Q$ tangent equations. Thus, if we have to perform $n_{\mathrm{CG}}$ steps of the method of conjugate gradients per Newton step, we should favor Algorithm 2.3, if

$$
\frac{\operatorname{dim} Q}{2} \leq n_{\mathrm{CG}}
$$

In Section 4, we will discuss a comparison of these two algorithms in the context of windowing.

\section{Discretization}

In this section, we discuss the discretization of the optimization problem (5). To this end, we use finite element method in time and space to discretize the state equation. This allows us to give a natural computable representation of the discrete gradient and Hessian. The use of exact discrete derivatives is important for the convergence of the optimization algorithms.

We discuss the corresponding (discrete) formulation of the auxiliary problems (dual, tangent and dual for Hessian) introduced in Section 2. The first 
subsection is devoted to semi-discretization in time by continuous Galerkin $(c G)$ and discontinuous Galerkin $(d G)$ methods. Subsection 3.2 deals with the space discretization of the semi-discrete problems arising from the time discretization. We also present the form of the required auxiliary equations for one concrete realization of the $\mathrm{cG}$ and the $\mathrm{dG}$ discretization respectively.

\subsection{Time Discretization}

To define a semi-discretization in time, let us partition the time interval $\bar{I}=$ $[0, T]$ as

$$
\bar{I}=\{0\} \cup I_{1} \cup I_{2} \cup \cdots \cup I_{M}
$$

with subintervals $I_{m}=\left(t_{m-1}, t_{m}\right]$ of size $k_{m}$ and time points

$$
0=t_{0}<t_{1}<\cdots<t_{M-1}<t_{M}=T \text {. }
$$

We define the parameter $k$ as a piecewise constant function by setting $\left.k\right|_{I_{m}}=$ $k_{m}$ for $m=1, \ldots, M$.

3.1.1 Discontinuous Galerkin (dG) Methods. We introduce for $r \in \mathbb{N}_{0}$ the discontinuous trial and test space

$$
X_{k}^{r}=\left\{v_{k} \in L^{2}(I, V)\left|v_{k}\right|_{I_{m}} \in P_{r}\left(I_{m}, V\right), m=1, \ldots, M, v_{k}(0) \in H\right\} .
$$

Here, we denote $P_{r}\left(I_{m}, V\right)$ the space of polynomial of degree $r$ defined on $I_{m}$ with values in $V$. Additionally, we will use the following notations for functions $v_{k} \in X_{k}^{r}$ :

$$
v_{k, m}^{+}=\lim _{t \rightarrow 0^{+}} v_{k}\left(t_{m}+t\right), \quad v_{k, m}^{-}=\lim _{t \rightarrow 0^{+}} v_{k}\left(t_{m}-t\right), \quad\left[v_{k}\right]_{m}=v_{k, m}^{+}-v_{k, m}^{-} .
$$


The dG discretization of the state equation (3) now reads: Find $u \in X_{k}^{r}$ such that

$$
\begin{gathered}
\sum_{m=1}^{M} \int_{I_{m}}\left\{\left(\partial_{t} u_{k}, \varphi\right)_{H}+a\left(q, u_{k}\right)(\varphi)\right\} d t+\sum_{m=1}^{M}\left(\left[u_{k}\right]_{m-1}, \varphi_{m-1}^{+}\right)_{H} \\
=\sum_{m=1}^{M} \int_{I_{m}}(f, \varphi)_{H} d t, \quad \forall \varphi \in X_{k}^{r} \\
u_{k, 0}^{-}=u_{0}(q) .
\end{gathered}
$$

For the analysis of the discontinuous finite element time discretization we refer to Estep and Larsson [10] and Eriksson, Johnson, and Thomée [9].

The corresponding semi-discrete optimization problem is given by:

$$
\text { Minimize } J\left(q, u_{k}\right) \text { subject to }(23),\left(q, u_{k}\right) \in Q \times X_{k}^{r},
$$

with the cost functional $J$ from (4).

Similar to the continuous case, we introduce a semi-discrete solution operator $S_{k}: Q_{k, 0} \rightarrow X_{k}^{r}$ such that $S_{k}(q)$ fulfills for $q \in Q_{k, 0}$ the semi-discrete state equation (23). As in Section 2, we define the semi-discrete reduced cost functional $j_{k}: Q_{k, 0} \rightarrow \mathbb{R}$ as

$$
j_{k}(q)=J\left(q, S_{k}(q)\right)
$$

and reformulate the optimization problem (24) as unconstrained problem:

$$
\text { Minimize } j_{k}(q), \quad q \in Q_{k, 0}
$$

To derive a representation of the derivatives of $j_{k}$, we define the semi-discrete Lagrangian $\mathcal{L}_{k}: Q \times X_{k}^{r} \times X_{k}^{r} \times H \rightarrow \mathbb{R}$, similar to the continuous case, as

$$
\begin{aligned}
\mathcal{L}_{k}\left(q, u_{k}, z_{k}, \tilde{z}_{k}\right)=J\left(q, u_{k}\right) & +\sum_{m=1}^{M} \int_{I_{m}}\left\{\left(f-\partial_{t} u_{k}, z_{k}\right)_{H}-a\left(q, u_{k}\right)\left(z_{k}\right)\right\} d t \\
& -\sum_{m=1}^{M}\left(\left[u_{k}\right]_{m-1}, z_{k, m-1}^{+}\right)_{H}-\left(u_{k, 0}^{-}-u_{0}(q), \tilde{z}_{k}\right)_{H}
\end{aligned}
$$

With these preliminaries, we obtain similar expressions for the three auxiliary equations in terms of the semi-discrete Lagrangian as stated in the section before. However, the derivation of the explicite representations for the auxiliary 
equations requires some care due to the special form of the Lagrangian $\mathcal{L}_{k}$ for the $\mathrm{dG}$ discretization:

- Dual Equation for $d G$ : For given $q \in Q_{k, 0}$ and $u_{k}=S_{k}(q) \in X_{k}^{r}$, find $\left(z_{k}, \tilde{z}_{k}\right) \in X_{k}^{r} \times H$ such that

$$
\begin{gathered}
\sum_{m=1}^{M} \int_{I_{m}}\left\{-\left(\varphi, \partial_{t} z_{k}\right)_{H}+a_{u}^{\prime}\left(q, u_{k}\right)\left(\varphi, z_{k}\right)\right\} d t-\sum_{m=1}^{M-1}\left(\varphi_{m}^{-},\left[z_{k}\right]_{m}\right)_{H} \\
+\left(\varphi_{M}^{-}, z_{k, M}^{-}\right)_{H}=\sum_{m=1}^{M} \int_{I_{m}} I^{\prime}\left(u_{k}\right)(\varphi) d t+K^{\prime}\left(u_{k, M}^{-}\right)\left(\varphi_{M}^{-}\right), \quad \forall \varphi \in X_{k}^{r}, \\
\tilde{z}_{k}=z_{k, 0}^{+} .
\end{gathered}
$$

- Tangent Equation for $d G$ : For $q \in Q_{k, 0}, u_{k}=S_{k}(q) \in X_{k}^{r}$, and a given direction $\delta q \in Q$, find $\delta u_{k} \in X_{k}^{r}$ such that

$$
\begin{gathered}
\sum_{m=1}^{M} \int_{I_{m}}\left\{\left(\partial_{t} \delta u_{k}, \varphi\right)_{H}+a_{u}^{\prime}\left(q, u_{k}\right)\left(\delta u_{k}, \varphi\right)\right\} d t+\sum_{m=1}^{M}\left(\left[\delta u_{k}\right]_{m-1}, \varphi_{m-1}^{+}\right)_{H} \\
=-\sum_{m=1}^{M} \int_{I_{m}} a_{q}^{\prime}\left(q, u_{k}\right)(\delta q, \varphi) d t, \quad \forall \varphi \in X_{k}^{r} \\
\delta u_{k, 0}^{-}=u_{0}^{\prime}(q)(\delta q) .
\end{gathered}
$$

- Dual for Hessian Equation for $d G$ : For given $q \in Q_{k, 0}, u_{k}=S_{k}(q) \in X_{k}^{r}$, $\left(z_{k}, \tilde{z}_{k}\right) \in X_{k}^{r} \times H$ the corresponding solution of the dual equation (25), and $\delta u_{k} \in X_{k}^{r}$ the solution of the tangent equation (26) for the given direction $\delta q$, find $\left(\delta z_{k}, \delta \tilde{z}_{k}\right) \in X_{k}^{r} \times H$ such that

$$
\begin{gathered}
\sum_{m=1}^{M} \int_{I_{m}}\left\{-\left(\varphi, \partial_{t} \delta z_{k}\right)_{H}+a_{u}^{\prime}\left(q, u_{k}\right)\left(\varphi, \delta z_{k}\right)\right\} d t-\sum_{m=1}^{M-1}\left(\varphi_{m}^{-},\left[\delta z_{k}\right]_{m}\right)_{H} \\
+\left(\varphi_{M}^{-}, \delta z_{k, M}^{-}\right)_{H}=-\sum_{m=1}^{M} \int_{I_{m}}\left\{a_{u u}^{\prime \prime}\left(q, u_{k}\right)\left(\delta u_{k}, \varphi, z_{k}\right)\right. \\
\left.+a_{q u}^{\prime \prime}\left(q, u_{k}\right)\left(\delta q, \varphi, z_{k}\right)\right\} d t+\sum_{m=1}^{M} \int_{I_{M}} I^{\prime \prime}\left(u_{k}\right)\left(\delta u_{k}, \varphi\right) d t \\
+K^{\prime \prime}\left(u_{k, M}^{-}\right)\left(\delta u_{k, M}^{-}, \varphi_{M}^{-}\right), \quad \forall \varphi \in X_{k}^{r}, \\
\delta \tilde{z}_{k}=\delta z_{k, 0}^{+} .
\end{gathered}
$$

As on the continuous level, the tangent equation can be obtained directly by calculating the derivatives of the Lagrangian, and for the dual equations, 
we additionally integrate by parts. But, since the test functions are piecewise polynomials, we can not separate the terms containing $\varphi_{M}^{-}$as we did it for the boundary terms in the continuous formulation before. However, because the support of $\varphi_{0}$ is just the point 0 , separation of the equation to determine $\tilde{z}_{k}$ or $\delta \tilde{z}_{k}$ is still possible.

Now, the representations from Theorem 2.1 and Theorem 2.2 can be translated to the semi-discrete level: We have

$$
\begin{aligned}
j_{k}^{\prime}(q)(\tau q) & =\alpha(q-\bar{q}, \tau q)_{Q} \\
& -\sum_{m=1}^{M} \int_{I_{m}} a_{q}^{\prime}\left(q, u_{k}\right)\left(\tau q, z_{k}\right) d t+\left(u_{0}^{\prime}(q)(\tau q), \tilde{z}_{k}\right)_{H}, \quad \forall \tau q \in Q,
\end{aligned}
$$

and, depending on whether we use version (a) or (b) of Theorem 2.2,

$$
\begin{aligned}
& j_{k}^{\prime \prime}(q)(\delta q, \tau q)=\alpha(\delta q, \tau q)_{Q} \\
& -\sum_{m=1}^{M} \int_{I_{m}}\left\{a_{q q}^{\prime \prime}\left(q, u_{k}\right)\left(\delta q, \tau q, z_{k}\right)+a_{u q}^{\prime \prime}\left(q, u_{k}\right)\left(\delta u_{k}, \tau q, z_{k}\right)+a_{q}^{\prime}\left(q, u_{k}\right)\left(\tau q, \delta z_{k}\right)\right\} d t \\
& +\left(u_{0}^{\prime}(q)(\tau q), \delta \tilde{z}_{k}\right)_{H}+\left(u_{0}^{\prime \prime}(q)(\delta q, \tau q), \tilde{z}_{k}\right)_{H}, \quad \forall \tau q \in Q, \quad(29)
\end{aligned}
$$

or

$$
\begin{aligned}
& j_{k}^{\prime \prime}(q)(\delta q, \tau q)=\alpha(\delta q, \tau q)_{Q}+\sum_{m=1}^{M} \int_{I_{m}} I_{u u}^{\prime \prime}\left(u_{k}\right)\left(\delta u_{k}, \tau u_{k}\right) d t \\
& -\sum_{m=1}^{M} \int_{I_{m}}\left\{a_{q q}^{\prime \prime}\left(q, u_{k}\right)\left(\delta q, \tau q, z_{k}\right)+a_{u q}^{\prime \prime}\left(q, u_{K}\right)\left(\delta u_{k}, \tau q, z_{k}\right)+a_{q u}^{\prime \prime}\left(q, u_{k}\right)\left(\delta q, \tau u_{k}, z_{k}\right)\right. \\
& \left.+a_{u u}^{\prime \prime}\left(q, u_{k}\right)\left(\delta u_{k}, \tau u_{k}, z_{k}\right)\right\} d t+K_{u u}^{\prime \prime}\left(u_{k}\right)\left(\delta u_{k}, \tau u_{k}\right) . \quad \text { (30) }
\end{aligned}
$$

3.1.2 Continuous Galerkin (cG) Methods. In this subsection, we discuss the time discretization by Galerkin methods with continuous trial functions and discontinuous test functions, the so called cG methods. For the test space, we use the space $X_{k}^{r}$ defined in (22), and additionally, we introduce a trial space given by:

$$
Y_{k}^{s}=\left\{v_{k} \in C(\bar{I}, V)\left|v_{k}\right|_{I_{m}} \in P_{s}\left(I_{m}, V\right), m=1, \ldots, M\right\} .
$$

To simplify the notation, we will use in this subsection the same symbols for the Lagrangian and the several solutions as in the subsection above for the $\mathrm{dG}$ 
discretization.

In virtue of these two spaces, we state the semi-discrete state equation in the $\mathrm{cG}$ context: Find $u_{k} \in Y_{k}^{s}$, such that

$$
\begin{aligned}
\int_{0}^{T}\left\{\left(\partial_{t} u_{k}, \varphi\right)_{H}+a\left(q, u_{k}\right)(\varphi)\right\} d t & =\int_{0}^{T}(f, \varphi)_{H} d t, \quad \forall \varphi \in X_{k}^{r} \\
u_{k}(0) & =u_{0}(q) .
\end{aligned}
$$

Similarly to the previous subsection, we define the semi-discrete optimization problem

$$
\text { Minimize } J\left(q, u_{k}\right) \text { subject to }(31),\left(q, u_{k}\right) \in Q \times Y_{k}^{s} \text {, }
$$

and the Lagrangian $\mathcal{L}_{k}: Q \times Y_{k}^{s} \times X_{k}^{r} \times H \rightarrow \mathbb{R}$ as

$$
\begin{aligned}
\mathcal{L}_{k}\left(q, u_{k}, z_{k}, \tilde{z}_{k}\right) & =J\left(q, u_{k}\right) \\
& +\int_{0}^{T}\left\{\left(f-\partial_{t} u_{k}, z_{k}\right)_{H}-a\left(q, u_{k}\right)\left(z_{k}\right)\right\} d t-\left(u_{k}(0)-u_{0}(q), \tilde{z}_{k}\right)_{H}
\end{aligned}
$$

Now, we can recall the process described in the previous subsection for the $\mathrm{dG}$ discretization to obtain the solution operator $S_{k}: Q_{k, 0} \rightarrow Y_{k}^{s}$, the reduced functional $j_{k}$, and the unconstrained optimization problem.

For the $\mathrm{cG}$ discretization, the three auxiliary equations read as follows:

- Dual Equation for $c G$ : For given $q \in Q_{k, 0}$ and $u_{k}=S_{k}(q) \in Y_{k}^{s}$, find $\left(z_{k}, \tilde{z}_{k}\right) \in X_{k}^{r} \times H$ such that

$$
\begin{gathered}
\sum_{m=1}^{M} \int_{I_{m}}\left\{-\left(\varphi, \partial_{t} z_{k}\right)_{H}+a_{u}^{\prime}\left(q, u_{k}\right)\left(\varphi, z_{k}\right)\right\} d t-\sum_{m=1}^{M-1}\left(\varphi\left(t_{m}\right),\left[z_{k}\right]_{m}\right)_{H} \\
+\left(\varphi(T), z_{k, M}^{-}\right)_{H}=\sum_{m=1}^{M} \int_{I_{m}} I^{\prime}\left(u_{k}\right)(\varphi) d t+K^{\prime}\left(u_{k}(T)\right)(\varphi(T)) \\
+\left(\varphi(0), z_{k, 0}^{+}-\tilde{z}_{k}\right)_{H}, \quad \forall \varphi \in Y_{k}^{s}
\end{gathered}
$$

- Tangent Equation for $c G$ : For $q \in Q_{k, 0}, u_{k}=S_{k}(q) \in Y_{k}^{s}$, and a given 
direction $\delta q \in Q$, find $\delta u_{k} \in Y_{k}^{s}$ such that

$$
\begin{gathered}
\int_{0}^{T}\left\{\left(\partial_{t} \delta u_{k}, \varphi\right)_{H}+a_{u}^{\prime}\left(q, u_{k}\right)\left(\delta u_{k}, \varphi\right)\right\} d t= \\
-\int_{0}^{T} a_{q}^{\prime}\left(q, u_{k}\right)(\delta q, \varphi) d t, \quad \forall \varphi \in X_{k}^{r} \\
\delta u_{k}(0)=u_{0}^{\prime}(q)(\delta q) .
\end{gathered}
$$

- Dual for Hessian Equation for $c G$ : For given $q \in Q_{k, 0}, u_{k}=S_{k}(q) \in Y_{k}^{s}$, $\left(z_{k}, \tilde{z}_{k}\right) \in X_{k}^{r} \times H$ the corresponding solution of the dual equation (33), and $\delta u_{k} \in Y_{k}^{s}$ the solution of the tangent equation (34) for the given direction $\delta q$, find $\left(\delta z_{k}, \delta \tilde{z}_{k}\right) \in X_{k}^{r} \times H$ such that

$$
\begin{aligned}
\sum_{m=1}^{M} \int_{I_{m}}\{ & \left.-\left(\varphi, \partial_{t} \delta z_{k}\right)_{H}+a_{u}^{\prime}\left(q, u_{k}\right)\left(\varphi, \delta z_{k}\right)\right\} d t-\sum_{m=1}^{M-1}\left(\varphi\left(t_{m}\right),\left[\delta z_{k}\right]_{m}\right)_{H} \\
& +\left(\varphi(T), \delta z_{k, M}^{-}\right)_{H}=-\sum_{m=1}^{M} \int_{I_{m}}\left\{a_{u u}^{\prime \prime}\left(q, u_{k}\right)\left(\delta u_{k}, \varphi, z_{k}\right)\right. \\
& \left.+a_{q u}^{\prime \prime}\left(q, u_{k}\right)\left(\delta q, \varphi, z_{k}\right)\right\} d t+\sum_{m=1}^{M} \int_{I_{M}} I^{\prime \prime}\left(u_{k}\right)\left(\delta u_{k}, \varphi\right) d t \\
& +K^{\prime \prime}\left(u_{k}\right)\left(\delta u_{k}(T), \varphi(T)\right)+\left(\varphi(0), z_{k, 0}^{+}-\tilde{z}_{k}\right)_{H}, \quad \forall \varphi \in Y_{k}^{s} .
\end{aligned}
$$

The derivation of the tangent equation (34) is straightforward and similar to the continuous case. However, the dual equation (33) and the dual for Hessian equation (35) contain jump terms such as $\left[z_{k}\right]_{m}$ or $\left[\delta z_{k}\right]_{m}$ due to the intervalwise integration by parts. As for the case of $\mathrm{dG}$ semi-discretization described in the previous subsection, the initial conditions can not be separated as in the continuous case, cf. (12) and (14). In contrast to the dG semi-discretization, we also can not separate the conditions to determine $\tilde{z}_{k}$ or $\delta \tilde{z}_{k}$ here, since for the $\mathrm{cG}$ methods the test functions of the dual equations are continuous, see the discussion for a concrete realization of the $\mathrm{cG}$ method in the next section.

Again, Theorem 2.1 and Theorem 2.2 are translated to the semi-discrete level by replacing the equations (12), (13) and (14) by the semi-discrete equations (33), (34) and (35). The representations of the derivatives of $j_{k}$ for the cG discretization have then the same form as in the dG case. Therefore, one should use formulas (28), (29) and (30), where $u_{k}, \delta u_{k}, z_{k}, \delta z_{k}, \tilde{z}_{k}$ and $\delta \tilde{z}_{k}$ are now determined by (31), (33), (34), and (35). 


\subsection{Space-time Discretization}

In this subsection, we first describe the finite element discretization in space. To this end, we consider two or three dimensional shape-regular meshes, see, e.g., Ciarlet [7]. A mesh consists of cells $K$, which constitute a non-overlapping cover of the computational domain $\Omega \subset \mathbb{R}^{d}, d \in\{2,3\}$. The corresponding mesh is denoted by $\mathcal{T}_{h}=\{K\}$, where we define the parameter $h$ as a cellwise constant function by setting $\left.h\right|_{K}=h_{K}$ with the diameter $h_{K}$ of the cell $K$.

On the mesh $\mathcal{T}_{h}$ we construct a finite element space $V_{h} \subset V$ in standard way:

$$
V_{h}=\left\{v \in V|v|_{K} \in \widetilde{Q}_{l}(K) \text { for } K \in \mathcal{T}_{h}\right\}
$$

Here, $\widetilde{Q}_{l}(K)$ consists of shape functions obtained via (bi-)linear transformations of functions in $Q_{l}(\widehat{K})$ defined on the reference cell $\widehat{K}=(0,1)^{2}$.

Now, the time-discretized schemes developed in the two previous subsections can be transfered to the full discretized level. For doing this, we use the spaces

$$
X_{h k}^{r}=\left\{v_{h k} \in L^{2}\left(I, V_{h}\right)\left|v_{h k}\right|_{I_{m}} \in P_{r}\left(I_{m}, V_{h}\right), m=1, \ldots, M, v_{h k}(0) \in V_{h}\right\}
$$

and

$$
Y_{h k}^{s}=\left\{v_{h k} \in C\left(\bar{I}, V_{h}\right)\left|v_{h k}\right|_{I_{m}} \in P_{s}\left(I_{m}, V_{h}\right), m=1, \ldots, M\right\}
$$

instead of $X_{k}^{r}$ and $Y_{k}^{s}$.

Remark 3.1 Often, by solving problems with complex dynamical behavior, it is desirable to use time-dependent meshes $\mathcal{T}_{h_{m}}$. Then, $h_{m}$ describes the mesh used in time interval $I_{m}$ and we can use the same definition of $X_{h_{m} k}^{r}$ as before, because of the discontinuity in time. Consequently, $\mathrm{dG}$ discretization is directly applicable to time-dependent space discretizations. The definition of $Y_{h_{m} k}^{s}$ requires more care due to the request for continuity in time. An approach overcoming this difficulty can be found in Becker [1].

In the sequel, we present one concrete time-stepping scheme for the $\mathrm{dG}$ and the $\mathrm{cG}$ discretization combined with the finite element space discretization. These schemes correspond to the implicit Euler scheme and the Crank-Nicolson scheme, respectively.

To obtain the standard implicit Euler scheme as a special case of dG discretization, we choose $r=0$ and approximate the integrals arising by the box rule. Furthermore, we define $U_{m}=\left.u_{h k}\right|_{I_{m}}, \Delta U_{m}=\left.\delta u_{h k}\right|_{I_{m}}, Z_{m}=\left.z_{h k}\right|_{I_{m}}$, and $\Delta Z_{m}=\left.z_{h k}\right|_{I_{m}}$ for $i=1, \ldots, M$, and $U_{0}=u_{h k, 0}^{-}, \Delta U_{0}=\delta u_{h k, 0}^{-}, Z_{0}=\tilde{z}_{h k}$, and 
$\Delta Z_{0}=\delta \tilde{z}_{h k}$. With this, we obtain the following schemes for the dG-discretized state and auxiliary equations, which should be fulfilled for all $\varphi \in V_{h}$ :

- State Equation for $d G$ :

○ $m=0$ :

$$
\left(U_{0}, \varphi\right)_{H}=\left(u_{0}(q), \varphi\right)_{H}
$$

$\circ m=1, \ldots, M$ :

$$
\left(U_{m}, \varphi\right)_{H}+k_{m} a\left(q, U_{m}\right)(\varphi)=\left(U_{m-1}, \varphi\right)_{H}+k_{m}\left(f\left(t_{m}\right), \varphi\right)_{H}
$$

- Dual Equation for $d G$ :

$\circ m=M$ :

$$
\left(\varphi, Z_{M}\right)_{H}+k_{M} a_{u}^{\prime}\left(q, U_{M}\right)\left(\varphi, Z_{M}\right)=K^{\prime}\left(U_{M}\right)(\varphi)+k_{M} I^{\prime}\left(U_{M}\right)(\varphi)
$$

○ $m=M-1, \ldots, 1$ :

$$
\left(\varphi, Z_{m}\right)_{H}+k_{m} a_{u}^{\prime}\left(q, U_{m}\right)\left(\varphi, Z_{m}\right)=\left(\varphi, Z_{m+1}\right)_{H}+k_{m} I^{\prime}\left(U_{m}\right)(\varphi)
$$

$\circ m=0$ :

$$
\left(\varphi, Z_{0}\right)_{H}=\left(\varphi, Z_{1}\right)_{H}
$$

- Tangent Equation for $d G$ :

○ $m=0$ :

$$
\left(\Delta U_{0}, \varphi\right)_{H}=\left(u_{0}^{\prime}(q)(\delta q), \varphi\right)_{H}
$$

$\circ m=1, \ldots, M$ :

$$
\left(\Delta U_{m}, \varphi\right)_{H}+k_{m} a_{u}^{\prime}\left(q, U_{m}\right)\left(\Delta U_{m}, \varphi\right)=\left(\Delta U_{m-1}, \varphi\right)_{H}-k_{m} a_{q}^{\prime}\left(q, U_{m}\right)(\delta q, \varphi)
$$

- Dual for Hessian Equation for $d G$ :

○ $m=M$ :

$$
\begin{aligned}
\left(\varphi, \Delta Z_{M}\right)_{H}+k_{M} a_{u}^{\prime}\left(q, U_{M}\right)\left(\varphi, \Delta Z_{M}\right)= \\
K^{\prime \prime}\left(U_{M}\right)\left(\Delta U_{M}, \varphi\right)+k_{M} I^{\prime \prime}\left(U_{M}\right)\left(\Delta U_{M}, \varphi\right) \\
-k_{M}\left\{a_{u u}^{\prime \prime}\left(q, U_{M}\right)\left(\Delta U_{M}, \varphi, Z_{M}\right)+a_{q u}^{\prime \prime}\left(q, U_{M}\right)\left(\delta q, \varphi, Z_{M}\right)\right\}
\end{aligned}
$$


$\circ m=M-1, \ldots, 1$ :

$$
\begin{aligned}
\left(\varphi, \Delta Z_{m}\right)_{H}+ & k_{m} a_{u}^{\prime}\left(q, U_{m}\right)\left(\varphi, \Delta Z_{m}\right)= \\
& \left(\varphi, \Delta Z_{m+1}\right)_{H}+k_{m} I^{\prime \prime}\left(U_{m}\right)\left(\Delta U_{m}, \varphi\right) \\
- & k_{m}\left\{a_{u u}^{\prime \prime}\left(q, U_{m}\right)\left(\Delta U_{m}, \varphi, Z_{m}\right)+a_{q u}^{\prime \prime}\left(q, U_{m}\right)\left(\delta q, \varphi, Z_{m}\right)\right\}
\end{aligned}
$$

$\circ m=0$ :

$$
\left(\varphi, \Delta Z_{0}\right)_{H}=\left(\varphi, \Delta Z_{1}\right)_{H}
$$

Remark 3.2 The implicit Euler scheme is known to be a first order strongly A-stable method. The resulting schemes for the auxiliary equations have basically the same structure and lead consequently to first order approximation, too. However, the precise a priori error analysis for the optimization problem requires more care and depends on the given structure of the problem under consideration.

The Crank-Nicolson scheme can be obtained in the context of cG discretization by choosing $r=0, s=1$ and approximating the integrals arising by the trapezoidal rule. Using the representation of the Crank-Nicolson scheme as a cG-scheme, allows us directly to give a concrete form of the auxiliary equations leading to the exact computation of the discrete gradient and Hessian.

We set $U_{m}=u_{h k}\left(t_{m}\right), \Delta U_{m}=\delta u_{h k}\left(t_{m}\right), Z_{m}=\left.z_{h k}\right|_{I_{m}}$, and $\Delta Z_{m}=\left.z_{h k}\right|_{I_{m}}$ for $i=1, \ldots, M$, and $U_{0}=u_{h k}(0), \Delta U_{0}=\delta u_{h k}(0), Z_{0}=\tilde{z}_{h k}$, and $\Delta Z_{0}=\delta \tilde{z}_{h k}$. With this, we obtain the following schemes for the cG-discretized state and auxiliary equations, which should be fulfilled for all $\varphi \in V_{h}$ :

- State Equation for $c G$ :

○ $m=0$ :

$$
\left(U_{0}, \varphi\right)_{H}=\left(u_{0}(q), \varphi\right)_{H}
$$

$\circ m=1, \ldots, M$ :

$$
\begin{aligned}
\left(U_{m}, \varphi\right)_{H}+ & \frac{k_{m}}{2} a\left(q, U_{m}\right)(\varphi)=\left(U_{m-1}, \varphi\right)_{H} \\
& -\frac{k_{m}}{2} a\left(q, U_{m-1}\right)(\varphi)+\frac{k_{m}}{2}\left\{\left(f\left(t_{m-1}\right), \varphi\right)_{H}+\left(f\left(t_{m}\right), \varphi\right)_{H}\right\}
\end{aligned}
$$

- Dual Equation for $c G$ : 
$\circ m=M$ :

$$
\left(\varphi, Z_{M}\right)_{H}+\frac{k_{M}}{2} a_{u}^{\prime}\left(q, U_{M}\right)\left(\varphi, Z_{M}\right)=K^{\prime}\left(U_{M}\right)(\varphi)+\frac{k_{M}}{2} I^{\prime}\left(U_{M}\right)(\varphi)
$$

$\circ m=M-1, \ldots, 1$ :

$$
\begin{aligned}
\left(\varphi, Z_{m}\right)_{H}+\frac{k_{m}}{2} a_{u}^{\prime}\left(q, U_{m}\right)\left(\varphi, Z_{m}\right)=\left(\varphi, Z_{m+1}\right)_{H} & \\
& \quad-\frac{k_{m+1}}{2} a_{u}^{\prime}\left(q, U_{m}\right)\left(\varphi, Z_{m+1}\right)+\frac{k_{m}+k_{m+1}}{2} I^{\prime}\left(U_{m}\right)(\varphi)
\end{aligned}
$$

$\circ m=0$ :

$$
\left(\varphi, Z_{0}\right)_{H}=\left(\varphi, Z_{1}\right)_{H}-\frac{k_{1}}{2} a_{u}^{\prime}\left(q, U_{0}\right)\left(\varphi, Z_{1}\right)+\frac{k_{1}}{2} I^{\prime}\left(U_{0}\right)(\varphi)
$$

- Tangent Equation for $c G$ :

○ $m=0$ :

$$
\left(\Delta U_{0}, \varphi\right)_{H}=\left(u_{0}^{\prime}(q)(\delta q), \varphi\right)_{H}
$$

$\circ m=1, \ldots, M$ :

$$
\begin{aligned}
&\left(\Delta U_{m}, \varphi\right)_{H}+\frac{k_{m}}{2} a_{u}^{\prime}\left(q, U_{m}\right)\left(\Delta U_{m}, \varphi\right)= \\
&\left(\Delta U_{m-1}, \varphi\right)_{H}-\frac{k_{m}}{2} a_{u}^{\prime}\left(q, U_{m-1}\right)\left(\Delta U_{m-1}, \varphi\right) \\
& \quad-\frac{k_{m}}{2}\left\{a_{q}^{\prime}\left(q, U_{m-1}\right)(\delta q, \varphi)+a_{q}^{\prime}\left(q, U_{m}\right)(\delta q, \varphi)\right\}
\end{aligned}
$$

- Dual for Hessian Equation for $c G$ :

○ $m=M$ :

$$
\begin{aligned}
\left(\varphi, \Delta Z_{M}\right)_{H}+ & \frac{k_{M}}{2} a_{u}^{\prime}\left(q, U_{M}\right)\left(\varphi, \Delta Z_{M}\right)= \\
& K^{\prime \prime}\left(U_{M}\right)\left(\Delta U_{M}, \varphi\right)+\frac{k_{M}}{2} I^{\prime \prime}\left(U_{M}\right)\left(\Delta U_{M}, \varphi\right) \\
- & \frac{k_{M}}{2}\left\{a_{u u}^{\prime \prime}\left(q, U_{M}\right)\left(\Delta U_{M}, \varphi, Z_{M}\right)+a_{q u}^{\prime \prime}\left(q, U_{M}\right)\left(\delta q, \varphi, Z_{M}\right)\right\}
\end{aligned}
$$


$\circ m=M-1, \ldots, 1$ :

$$
\begin{aligned}
\left(\varphi, \Delta Z_{m}\right)_{H}+\frac{k_{m}}{2} a_{u}^{\prime}\left(q, U_{m}\right)\left(\varphi, \Delta Z_{m}\right)=\left(\varphi, \Delta Z_{m+1}\right)_{H} \\
-\frac{k_{m+1}}{2} a_{u}^{\prime}\left(q, U_{m}\right)\left(\varphi, \Delta Z_{m+1}\right)+\frac{k_{m}+k_{m+1}}{2} I^{\prime \prime}\left(U_{m}\right)\left(\Delta U_{m}, \varphi\right) \\
-\frac{k_{m}}{2}\left\{a_{u u}^{\prime \prime}\left(q, U_{m}\right)\left(\Delta U_{m}, \varphi, Z_{m}\right)+a_{q u}^{\prime \prime}\left(q, U_{m}\right)\left(\delta q, \varphi, Z_{m}\right)\right\} \\
-\frac{k_{m+1}}{2}\left\{a_{u u}^{\prime \prime}\left(q, U_{m}\right)\left(\Delta U_{m}, \varphi, Z_{m+1}\right)+a_{q u}^{\prime \prime}\left(q, U_{m}\right)\left(\delta q, \varphi, Z_{m+1}\right)\right\}
\end{aligned}
$$

$\circ m=0$ :

$$
\begin{aligned}
\left(\varphi, \Delta Z_{0}\right)_{H}=( & \left., \Delta Z_{1}\right)_{H}-\frac{k_{1}}{2} a_{u}^{\prime}\left(q, U_{0}\right)\left(\varphi, Z_{1}\right)+\frac{k_{1}}{2} I^{\prime \prime}\left(U_{0}\right)\left(\Delta U_{0}, \varphi\right) \\
& -\frac{k_{1}}{2}\left\{a_{u u}^{\prime \prime}\left(q, U_{0}\right)\left(\Delta U_{0}, \varphi, Z_{1}\right)+a_{q u}^{\prime \prime}\left(q, U_{0}\right)\left(\delta q, \varphi, Z_{1}\right)\right\}
\end{aligned}
$$

The resulting Crank-Nicolson scheme is known to be of second order. However, in contrast to the implicit Euler scheme, this method does not possess the strong A-stability property. The structure of the time-steps for the dual and the dual for Hessian equations is quite unusual. In the first and in the last steps, "half-steps" occur, and in the other steps, terms containing the sizes of two neighboring time intervals $k_{m}$ and $k_{m+1}$ appear. This complicates the a priori error analysis for the dual scheme, which can be found in Becker [1].

\section{Windowing}

When computing the gradient of the reduced cost functional as described in the algorithms in Section 2, we need to have access to the solution of the state equation at all points in space and time while computing the dual equation. Similarly, we need the solution of the state, tangent, and dual equations to solve the dual for Hessian equation when computing matrix-vector products with the Hessian of the reduced functional. For large problems, especially in three dimensions, storing all the necessary data might be impossible. However, there are techniques to reduce the storage requirements drastically, known as checkpointing techniques.

In this section, we present an approach, which relies on ideas from Berggren, Glowinski, and Lions [5]. In the sequel, we extend these ideas to obtain two concrete algorithms and present an extension to apply the algorithms to the 
whole optimization loops showed in Section 2. Due to its structure, we call this approach Multi-Level Windowing.

\subsection{The Abstract Algorithm}

First, we consider the following abstract setting: Let two time stepping schemes be given:

$$
\begin{array}{rlrl}
\mathrm{x}_{m-1} & \mapsto \mathrm{x}_{m}, & & \text { for } m=1, \ldots, M, \\
\left(\mathrm{y}_{m+1}, \mathrm{x}_{m}\right) & \mapsto \mathrm{y}_{m}, & \text { for } m=M-1, \ldots, 0,
\end{array}
$$

together with a given initial value $\mathrm{x}_{0}$ and the mapping $\mathrm{x}_{M} \mapsto \mathrm{y}_{M}$. All time stepping schemes given for $\mathrm{dG}$ and $\mathrm{cG}$ discretization in the previous section are concrete realizations of these abstract schemes.

Additionally, we assume that the solutions $\mathrm{x}_{m}$ as well as $\mathrm{y}_{m}$ require for all $m=0, \ldots, M$ the same amount of storage. However, if this is not the case, the windowing technique presented in the sequel can be applied to clusters of time steps similar in size instead of single time steps. Such clustering is, e.g., important by using dynamical meshes, since in this case, the amount of storage for a solution $x_{m}$ depends on the current mesh.

The trivial approach to perform the forward and backwards iterations is to compute and store the whole forward solution $\left\{\mathrm{x}_{m}\right\}_{m=0}^{M}$, and use these values to compute the backwards solution $\left\{\mathrm{y}_{m}\right\}_{m=0}^{M}$. The required amount of storage $S_{0}$ in terms of the size of one forward solution $\mathrm{x}_{m}$ to do this is $S_{0}=M+1$. The number of forward steps $W_{0}$ necessary to compute the whole backwards solution is $W_{0}=M$.

The aim of the following windowing algorithms is to reduce the needed storage by performing some additional forward steps. To introduce the windowing, we additionally assume that we can factorize the number of given time steps $M$ as $M=P Q$ with positive integers $P$ and $Q$. With this, we can separate the set of time points $\{0, \ldots, M\}$ in $P$ slices each containing $Q-1$ time steps and $P+1$ sets containing one element as

$$
\begin{aligned}
\{0, \ldots, M\}=\{0\} & \cup\{1, \ldots, Q-1\} \cup\{Q\} \cup \cdots \\
& \cdots \cup\{(P-1) Q\} \cup\{(P-1) Q+1, \ldots, P Q-1\} \cup\{P Q\} .
\end{aligned}
$$

The algorithm now works as follows: First, we compute the forward solution $\mathrm{x}_{m}$ for $m=1, \ldots, M$ and store the $P+1$ samples $\left\{\mathrm{x}_{Q l}\right\}_{l=0}^{P}$. Additionally, we store the $Q-1$ values of $\mathrm{x}$ in the last slice. Now, we have the necessary information on $\mathrm{x}$ to compute $\mathrm{y}_{m}$ for $m=M, \ldots,(P-1) Q+1$. Thus, the 
values of $\mathrm{x}$ in the last slice are not longer needed. We can replace them with the values of $\mathrm{x}$ in the next-last slice, which we can directly compute using the time stepping scheme since we stored the value $\mathrm{x}_{(P-2) Q}$ in the first run. Thereby, we can compute $\mathrm{y}_{m}$ for $m=(P-1) Q, \ldots,(P-2) Q+1$. This can now be done iteratively till we have computed y in the first slice and finally obtain the value $\mathrm{y}_{0}$. This so called One-Level Windowing is presented on detail in Algorithm 4.1.

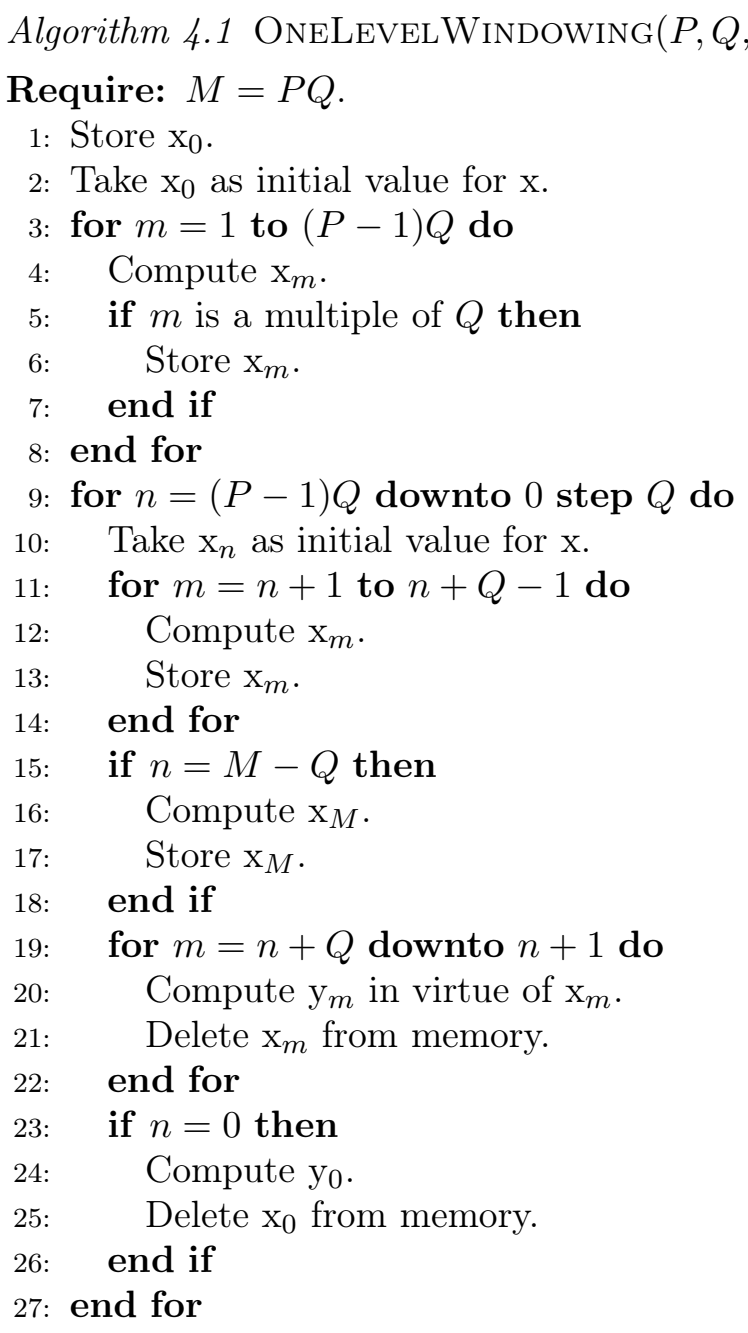

During the Execution of Algorithm 4.1, the needed amount of memory is not exceeding $(P+1)+(Q-1)$ forward solutions. Each of the $\mathrm{y}_{m}$ 's is computed exactly once, so we need $M$ solving steps to obtain the whole solution y. To compute the necessary values of $\mathrm{x}_{m}$, we have to solve $M+(P-1)(Q-1)$ forward steps, since we have to compute each of the values of $\mathrm{x}$ in the first 
$P-1$ slices. We summarize:

$$
S_{1}(P, Q)=P+Q, \quad W_{1}(P, Q)=2 M-P-Q+1,
$$

where again $S_{1}$ denotes the required amount of memory in terms of the size of one forward solution and $W_{1}$ the number of time steps to provide the forward solution $\mathrm{x}$ needed to compute the whole backwards solution $\mathrm{y}$.

Here, the subscript 1 suggests that we can extend this approach to factorizations of $M$ in $L+1$ factors for $L \in \mathbb{N}$. This extension can be obtained via the following inductive argumentation: Assuming $M=M_{0} M_{1} \cdots M_{L}$ with positive integers $M_{l}$, we can apply the algorithm described above to the factorization $M=P Q$ with $P=M_{0}$ and $Q=M_{1} M_{2} \cdots M_{L}$, and then recursively to each of the $P$ slices. This so called Multi-Level Windowing is described in Algorithm 4.2. It has to be started with the call MultiLevelWindowING $\left(0,0, L, M_{0}, M_{1}, \ldots, M_{L}, M\right)$. Of course, there holds by construction

$$
\begin{aligned}
\operatorname{OneLevelWindowing}(P, Q, M) & \\
& =\operatorname{MultiLevelWindowing}(0,0,1, P, Q, M) .
\end{aligned}
$$

Algorithm 4.2 MultiLevelWindowing $\left(s, l, L, M_{0}, M_{1}, \ldots, M_{L}, M\right)$ :

Require: $M=M_{0} M_{1} \cdots M_{L}$.

1: Set $P=M_{l}$ and $Q=M_{l+1} \cdots M_{L}$.

if $l=0$ and $s=0$ then

$$
\text { Store } \mathrm{x}_{0} \text {. }
$$

end if

: Take $\mathrm{x}_{s}$ as initial value for $\mathrm{x}$.

for $m=1$ to $(P-1) Q$ do

Compute $\mathrm{x}_{s+m}$.

if $m$ is a multiple of $Q$ then

Store $\mathrm{x}_{s+m}$.

end if

: end for

for $n=(P-1) Q$ downto 0 step $Q$ do

if $l+1<L$ then

Call MultiLevelWindowing $\left(s+n, l+1, L, M_{0}, M_{1}, \ldots, M_{L}, M\right)$. else

Take $\mathrm{x}_{s+n}$ as initial value for $\mathrm{x}$.

for $m=n+1$ to $n+Q-1$ do

Compute $\mathrm{x}_{s+m}$.

Store $\mathrm{x}_{s+m}$.

end for 


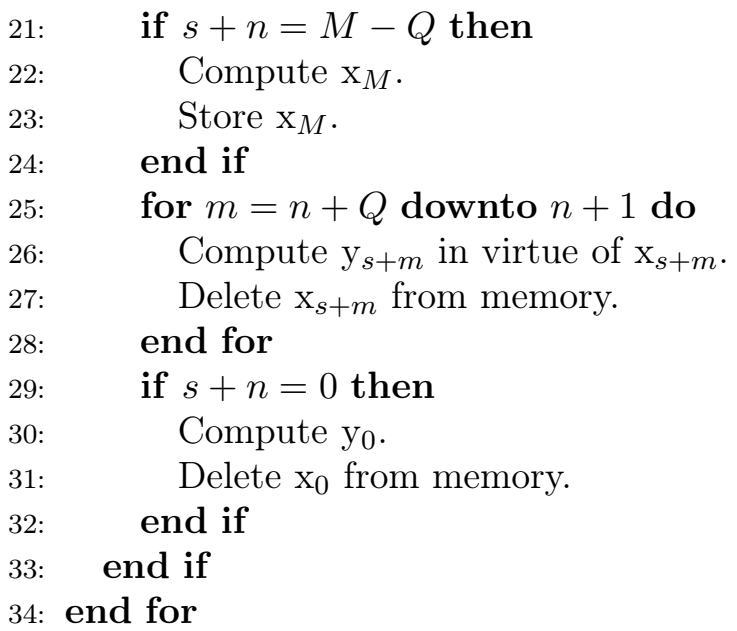

Remark 4.1 The presented approach can be extended to cases where a suitable factorization $M=M_{0} M_{1} \cdots M_{L}$ does not exist. We then consider a representation of $M$ as $M=\left(M_{0}-1\right) Q_{0}+R_{0}$ with positive integers $M_{0}, Q_{0}$ and $R_{0}$ with $Q_{0} \leq R_{0}<2 Q_{0}$ and apply this idea recursively to the generated subintervals of length $Q_{0}$ or $R_{0}$. This can easily be done, since by construction, the reminder interval of length $R_{0}$ has at least the same length as the regular subintervals.

In the following theorem, we calculate the necessary amount of storage and the number of needed forward steps to perform the Multi-Level Windowing described in Algorithm 4.2 for a given factorization $M=M_{0} M_{1} \cdots M_{L}$ of length $L+1$ :

TheOREM 4.1 For given $L \in \mathbb{N}_{0}$ and a factorization of the number of time steps $M$ as $M=M_{0} M_{1} \cdots M_{L}$ with $M_{l} \in \mathbb{N}$, the required amount of memory in the Multi-Level Windowing to perform all backwards solution steps is

$$
S_{L}\left(M_{0}, M_{1}, \ldots, M_{L}\right)=\sum_{l=0}^{L}\left(M_{l}-1\right)+2 .
$$

To achieve this storage reduction, the number of performed forward steps enhances to

$$
W_{L}\left(M_{0}, M_{1}, \ldots, M_{L}\right)=(L+1) M-\sum_{l=0}^{L} \frac{M}{M_{l}}+1
$$

Proof We prove the theorem by mathematical induction:

- $L=0$ : Here we use the trivial approach where the entire forward solution 
$\mathrm{x}$ is saved. As considered in the beginning of this subsection, we then have $S_{0}(M)=M+1$ and $W_{0}(M)=M$.

- $L-1 \rightsquigarrow L$ : We consider the factorization $M=M_{0} M_{1} \cdots M_{L-2}\left(M_{L-1} M_{L}\right)$ of length $L$ additionally to the given one of length $L+1$. Then we obtain in the same way as for the One-Level Windowing, where we reduce the storage mainly from $P Q-1$ to $(P-1)+(Q-1)$,

$$
\begin{aligned}
& S_{L}\left(M_{0}, M_{1}, \ldots, M_{L-1}, M_{L}\right) \\
= & S_{L-1}\left(M_{0}, M_{1}, \ldots, M_{L-1} M_{L}\right)-\left(M_{L-1} M_{L}-1\right)+\left(M_{L-1}-1\right)+\left(M_{L}-1\right) .
\end{aligned}
$$

In virtue of the induction hypothesis for $S_{L-1}$, it follows

$$
\begin{aligned}
S_{L}\left(M_{0}, M_{1}, \ldots, M_{L-1}, M_{L}\right) & =\sum_{l=0}^{L-2}\left(M_{l}-1\right)+\left(M_{L-1}-1\right)+\left(M_{L}-1\right)+2 \\
& =\sum_{l=0}^{L}\left(M_{l}-1\right)+2 .
\end{aligned}
$$

Now, we prove the assertion for $W_{L}$. For this, we justify the equality

$$
\begin{aligned}
& W_{L}\left(M_{0}, M_{1}, \ldots, M_{L-1}, M_{L}\right) \\
& \quad=W_{L-1}\left(M_{0}, M_{1}, \ldots, M_{L-1} M_{L}\right)+\frac{M}{M_{L-1} M_{L}}\left(M_{L-1}-1\right)\left(M_{L}-1\right) .
\end{aligned}
$$

This follows directly from the fact that we divide each of the $\frac{M}{M_{L-1} M_{L}}$ slices $\left\{s+1, \ldots, s+M_{L-1} M_{L}-1\right\}$ of length $M_{L-1} M_{L}-1$ as

$$
\begin{gathered}
\left\{s+1, \ldots, s+M_{L-1} M_{L}-1\right\}=\left\{s+1, \ldots, s+M_{L}-1\right\} \cup\left\{s+M_{L}\right\} \cup \cdots \\
\cdots \cup\left\{s+\left(M_{L-1}-1\right) M_{L}\right\} \cup\left\{s+\left(M_{L-1}-1\right) M_{L}+1, \ldots, s+M_{L-1} M_{L}-1\right\} .
\end{gathered}
$$

Since we just need to compute the forward solution in the first $M_{L-1}-1$ subslices when we change from the factorization of length $L$ to the one of length $L+1$, the additional work is

$$
\frac{M}{M_{L-1} M_{L}}\left(M_{L-1}-1\right)\left(M_{L}-1\right)
$$


as stated. Then we obtain in virtue of the induction hypothesis for $W_{L-1}$

$$
\begin{aligned}
W_{L}\left(M_{0}, M_{1}, \ldots, M_{L-1}, M_{L}\right) & =L M+M-\sum_{l=0}^{L-2} \frac{M}{M_{l}}-\frac{M}{M_{L-1}}-\frac{M}{M_{L}}+1 \\
& =(L+1) M-\sum_{l=0}^{L} \frac{M}{M_{l}}+1 .
\end{aligned}
$$

If $M^{\frac{1}{L+1}} \in \mathbb{N}$, the minimum of $\widetilde{S}_{L}$ of all possible factorizations of length $L+1$ is

$$
\widetilde{S}_{L}=S_{L}\left(M^{\frac{1}{L+1}}, \ldots, M^{\frac{1}{L+1}}\right)=(L+1)\left(M^{\frac{1}{L+1}}-1\right)+2 .
$$

The numbers of forward steps for the memory-optimal factorization then results in

$$
\widetilde{W}_{L}=W_{L}\left(M^{\frac{1}{L+1}}, \ldots, M^{\frac{1}{L+1}}\right)=(L+1)\left(M-M^{\frac{L}{L+1}}\right)+1 .
$$

If we choose $L \approx \log _{2} M$, then we obtain for the optimal factorization from above logarithmic growth of the necessary amount of storage:

$$
\widetilde{S}_{L}=O\left(\log _{2} M\right), \quad \widetilde{W}_{L}=O\left(M \log _{2} M\right) .
$$

Remark 4.2 If we consider time stepping schemes which depend not only on the immediate but on $p$ predecessors, i.e.

$$
\left(\mathrm{x}_{m-p}, \mathrm{x}_{m-p+1}, \ldots, \mathrm{x}_{m-1}\right) \mapsto \mathrm{x}_{m}, \quad \text { for } m=p, \ldots, M
$$

with given initial values $\mathrm{x}_{0}, \mathrm{x}_{1}, \ldots, \mathrm{x}_{p-1}$, the presented windowing approach can not be used directly. One possibility to extend this concept to such cases is to save $p$ values of $\mathrm{x}$ instead of one at each checkpoint. Then, during the backwards run, we will always have access to the necessary information on $\mathrm{x}$ to compute $\mathrm{y}$.

\subsection{Application to Optimization}

In this subsection, we consider the Multi-Level Windowing, described in the previous subsection, in the context of nonstationary optimization. We give a detailed estimate of the number of steps and the amount of memory required to perform one Newton step for a given number of levels $L \in \mathbb{N}$. For 
brevity, we will just write $W_{L}$ and $S_{L}$ instead of $W_{L}\left(M_{0}, M_{1}, \ldots, M_{L}\right)$ and $S_{L}\left(M_{0}, M_{1}, \ldots, M_{L}\right)$.

4.2.1 Optimization Loop without Building up the Hessian. First, we treat the variant of the optimization algorithms, which does not build up the entire Hessian of the reduced functional and is given in Algorithm 2.1. As stated in this algorithm, it is necessary to compute the value of the reduced functional and the gradient one time per Newton step. To apply the derived windowing techniques, we set $\mathrm{x}=u, \mathrm{y}=z$ and note, that Algorithm 4.2 can easily be extended to compute the necessary terms for evaluating the functional and the gradient during the forward or backwards computation, respectively. Thus, the total number of times steps needed to do this, is $W^{\text {grad }}=W_{L}+M$. The required amount of memory is $S^{\text {grad }}=S_{L}$.

Additionally to the gradient, we need to compute one matrix-vector product of the Hessian times a given vector in each of the $n_{\mathrm{CG}}$ steps of the conjugate gradient method. This is done as described in Algorithm 2.2. For avoiding the storage of $u$ or $z$ in all time steps, we have to compute $u, \delta u, z$, and $\delta z$ again in every $\mathrm{CG}$ step. Consequently, we set here $\mathrm{x}=(u, \delta u)$ and $\mathrm{y}=(z, \delta z)$. We obtain $W^{\text {hess }}=2\left(W_{L}+M\right)$ and $S^{\text {hess }}=2 S_{L}$.

In total we achieve

$$
\begin{aligned}
W^{(1)} & =W^{\text {grad }}+n_{\mathrm{CG}} W^{\text {hess }}=\left(1+2 n_{\mathrm{CG}}\right)\left(W_{L}+M\right), \\
S^{(1)} & =\max \left(S^{\text {grad }}, S^{\text {hess }}\right)=2 S_{L} .
\end{aligned}
$$

Remark 4.3 The windowing algorithm 4.2 can be modified to reduce the necessary forward steps under acceptance of increasing the needed amount of storage as follows: We do not to delete $u$ while computing $z$ at the points where $u$ is saved before starting the computation of $z$. Additionally, we save $z$ at these checkpoints. These saved values of $u$ and $z$ can be used to reduce the necessary number of forward steps to provide the values of $u$ and $\delta u$ for computing one matrix-vector product with the Hessian. Of course, when saving additional samples of $u$ and $z$, the needed amount of storage increases. For one Newton step we obtain the total work $\widetilde{W}^{(1)}$ and storage $\widetilde{S}^{(1)}$ as

$$
\widetilde{W}^{(1)}=W^{(1)}-2 n_{\mathrm{CG}} \min \left(S_{L}, M\right) \quad \text { and } \quad \widetilde{S}^{(1)}=S^{(1)}+2 S_{L}-M_{0}-2
$$

This modified algorithm includes the case of not using windowing for $L=0$, while the original algorithm also for $L=0$ deletes $u$ during the computation of $z$. 
4.2.2 Optimization Loop with Building up the Hessian. For using Algorithm 2.3, it is necessary to compute $u, \delta u_{i}(i=1, \ldots, \operatorname{dim} Q)$, and $z$. Again, the evaluation of the reduced functional is done during the first forward computation, and the evaluation of the gradient and the Hessian is done during the computation of $z$. So, we set $\mathrm{x}=\left(u, \delta u_{1}, \delta u_{2}, \ldots, \delta u_{\operatorname{dim} Q}\right)$ and $\mathrm{y}=z$. The required number of steps and the needed amount of memory are

$$
W^{(2)}=(1+\operatorname{dim} Q) W_{L}+M \quad \text { and } \quad S^{(2)}=(1+\operatorname{dim} Q) S_{L} .
$$

Remark 4.4 If we apply globalization techniques as line search to one of the presented optimization algorithms, we have to compute the solution of the state equation and the value of the cost functional several times without computing the gradient or the Hessian. The direct approach for doing this, is to compute the state solution, evaluate it and delete it afterwards. This might be not optimal, since for the following computation of the gradient (and the Hessian) via windowing, the needful preparations are not done. So, the better way of doing this is to run Algorithm 4.2 until line 23, and break so after completing the forward solution. If after that, the value of the gradient is needed, it is possible to restart directly on line 25 with the computation of the backwards solutions. If we consider the version presented in the actual subsection with building up the Hessian, we have to compute the tangent solutions in an extra forward run in which we can also use the saved values of the state solution.

\subsubsection{Comparison of the Two Variants of the Optimization Algorithm.} For $\operatorname{dim} Q \geq 1$, we obtain directly $S^{(2)} \geq S^{(1)}$. The relation between $W^{(1)}$ and $W^{(2)}$ depends on the factorization of $M$. A simple calculation leads to the following condition:

$$
W^{(2)} \leq W^{(1)} \Longleftrightarrow \frac{\operatorname{dim} Q}{2} \leq n_{\mathrm{CG}}\left(1+\frac{M}{W_{L}}\right) .
$$

If we choose $L$ such that $W_{L} \approx M \log _{2} M$, we can express the condition above just in terms of $M$ as

$$
W^{(2)} \lesssim W^{(1)} \Longleftrightarrow \quad \frac{\operatorname{dim} Q}{2} \lesssim n_{\mathrm{CG}}\left(1+\frac{1}{\log _{2} M}\right) .
$$

This means, that even thought the required memory for the second algorithm with building up the Hessian is greater, this algorithm needs only then fewer steps than the first one, if the necessary numbers of CG steps performed in each Newton step is greater than half of the dimension of $Q$ times a factor depending logarithmic on the number of time steps $M$. 


\section{$5 \quad$ Numerical Results}

In this last section, we present some illustrative numerical examples. Throughout, the spatial discretization is done with piecewise bilinear/trilinear finite elements on quadrilateral or hexahedral cells in two respectively three dimensions. The resulting nonlinear state equations are solved by Newton's method, whereas the linear sub-problems are treated by a multigrid method. For time discretization, we consider the variants of the $\mathrm{cG}$ and $\mathrm{dG}$ methods which we have presented in Section 3. Throughout this section, we only present results using the variant of the optimization loop building up the entire Hessian, described in Algorithm 2.3 since the results of the variant without building up the Hessian are mainly the same.

All computations are done based on the software packages RoDoBo [4] and Gascoigne [2]. To depict the computed solutions, the visualization software VisuSimple [3] was used.

We consider the following two example problems on the space-time domain $\Omega \times(0, T)$ with $T=1$.

- Example 1: In the first example we, discuss an optimal control problem with terminal observation, where the control variable enters the initial condition of the (nonlinear) state equation. We choose $\Omega=(0,1)^{3} \subset \mathbb{R}^{3}$ and pose the state equation as

$$
\begin{aligned}
& \partial_{t} u-\nu \Delta u+u^{2}=0 \text { in } \Omega \times(0, T), \\
& \partial_{n} u=0 \text { on } \partial \Omega \times(0, T), \\
& u(0, \cdot)=g_{0}+\sum_{i=1}^{8} g_{i} q_{i} \quad \text { on } \Omega,
\end{aligned}
$$

where $\nu=0.1, g_{0}=\left(1-2\left\|x-\bar{x}_{0}\right\|\right)^{30}$ with $\bar{x}_{0}=(0.5,0.5,0.5)^{T}$ and $g_{i}=$ $\left(1-0.5\left\|x-\bar{x}_{i}\right\|\right)^{30}$ with $\bar{x}_{i} \in\{0.2,0.8\}^{3}$ for $i=1, \ldots, 8$ are given.

For an additionally given reference solution

$$
\bar{u}_{T}(x)=\frac{3+x_{1}+x_{2}+x_{3}}{6}, \quad x=\left(x_{1}, x_{2}, x_{3}\right)^{T},
$$

the optimization problem now reads as:

Minimize $\frac{1}{2} \int_{\Omega}\left(u(T, \cdot)-\bar{u}_{T}\right)^{2} d x+\frac{\alpha}{2}\|q\|_{Q}$ subject to $(36),(q, u) \in Q \times X$,

where $Q=\mathbb{R}^{8}$ and $X$ is chosen in virtue of (2) with $V=H^{1}(\Omega)$ and 
$H=L^{2}(\Omega)$. The regularization parameter $\alpha$ is set to $10^{-4}$.

- Example 2: In the second example, we choose $\Omega=(0,1)^{2} \subset \mathbb{R}^{2}$ and consider a parameter estimation problem with the state equation given by

$$
\begin{array}{rlrl}
\partial_{t} u-\nu \Delta u+q_{1} \partial_{1} u+q_{2} \partial_{2} u & =2+\sin (10 \pi t) \quad \text { in } \Omega \times(0, T), \\
u & =0 & & \text { on } \partial \Omega \times(0, T), \\
u(0, \cdot) & =0 & & \text { on } \Omega,
\end{array}
$$

where we again set $\nu=0.1$.

We assume to be given measurements $\bar{u}_{T, 1}, \ldots, \bar{u}_{T, 5} \in \mathbb{R}$ of the point values $u\left(T, p_{i}\right)$ for five different measurement points $p_{i} \in \Omega$. The unknown parameters $\left(q_{1}, q_{2}\right) \in Q=\mathbb{R}^{2}$ are estimated using a least squares approach resulting in the following optimization problem:

$$
\text { Minimize } \frac{1}{2} \sum_{i=1}^{5}\left(u\left(T, p_{i}\right)-\bar{u}_{T, i}\right)^{2} \text { subject to }(37),(q, u) \in Q \times X \text {. }
$$

The consideration of point measurements does not fulfill the assumption on the cost functional in (4), since the point evaluation is not bounded as a functional on $H=L^{2}(\Omega)$. Therefore, the point functionals here may be understood as regularized functionals defined on $L^{2}(\Omega)$. For an a priori error analysis of an elliptic parameter identification problems with pointwise measurements we refer to Rannacher and Vexler [19].

\subsection{Validation of the Compututation of Derivatives}

To verify the computation of the gradient $\nabla j_{h k}$ and the Hessian $\nabla^{2} j_{h k}$ of the reduced cost functional, we consider the first and second difference quotients

$$
\begin{gathered}
\frac{j_{h k}(q+\varepsilon \delta q)-j_{h k}(q-\varepsilon \delta q)}{2 \varepsilon}=\left(\nabla j_{h k}, \delta q\right)+e_{1}, \\
\frac{j_{h k}(q+\varepsilon \delta q)-2 j_{h k}(q)+j_{h k}(q-\varepsilon \delta q)}{\varepsilon^{2}}=\left(\delta q, \nabla^{2} j_{h k} \delta q\right)+e_{2} .
\end{gathered}
$$

We obtain using standard convergence and stability analysis the concrete form of the errors $e_{1}$ and $e_{2}$ as

$$
e_{1} \approx c_{1} \varepsilon^{2} \nabla^{3} j_{h k}\left(\xi_{1}\right)+c_{2} \varepsilon^{-1}, \quad e_{2} \approx c_{3} \varepsilon^{2} \nabla^{4} j_{h k}\left(\xi_{2}\right)+c_{4} \varepsilon^{-2},
$$

where $\xi_{1}, \xi_{2} \in(q-\varepsilon \delta q, q+\varepsilon \delta q)$ is an intermediate point and the constants $c_{i}$ do not depend on $\varepsilon$. 
The Tables 1 and 2 show the errors between the values of the derivatives computed by use of the difference quotients above and by use of the approach presented in the Sections 2 and 3, for the considered examples. The values of these errors and the orders of convergence of the reduction of these errors for $\varepsilon \rightarrow 0$ are given in the Tables 1 and 2 . Note, that the values of the derivatives computed via the approach based on the ideas presented in Section 2 do not depend on $\varepsilon$.

The content of these tables does not considerably depend on the discretization parameters $h$ and $k$, so we have the exact discrete derivatives also on coarse meshes or when using large time steps.

\begin{tabular}{|c|c|c|c|c|c|c|c|c|}
\hline \multirow[b]{3}{*}{$\varepsilon$} & \multicolumn{4}{|c|}{ Discontinuous Galerkin } & \multicolumn{4}{|c|}{ Continuous Galerkin } \\
\hline & \multicolumn{2}{|c|}{ Gradient } & \multicolumn{2}{|c|}{ Hessian } & \multicolumn{2}{|c|}{ Gradient } & \multicolumn{2}{|c|}{ Hessian } \\
\hline & $e_{1}$ & Conv. & $e_{2}$ & Conv. & $e_{1}$ & Conv. & $e_{2}$ & Conv. \\
\hline $1.0 \mathrm{e}-00$ & $8.56 \mathrm{e}-01$ & - & $6.72 \mathrm{e}-01$ & - & $7.96 \mathrm{e}-01$ & - & $5.97 \mathrm{e}-01$ & - \\
\hline $1.0 \mathrm{e}-01$ & $5.37 \mathrm{e}-03$ & 2.20 & $4.32 \mathrm{e}-03$ & 2.19 & $5.28 \mathrm{e}-03$ & 2.17 & $4.08 \mathrm{e}-03$ & 2.16 \\
\hline $1.0 \mathrm{e}-02$ & $5.35 \mathrm{e}-05$ & 2.00 & $4.27 \mathrm{e}-05$ & 2.00 & $5.26 \mathrm{e}-05$ & 2.00 & $4.05 \mathrm{e}-05$ & 2.00 \\
\hline $1.0 \mathrm{e}-03$ & $5.34 \mathrm{e}-07$ & 2.00 & $3.28 \mathrm{e}-05$ & 0.11 & $5.26 \mathrm{e}-07$ & 2.00 & $3.27 \mathrm{e}-05$ & 0.09 \\
\hline $1.0 \mathrm{e}-04$ & $5.30 \mathrm{e}-09$ & 2.00 & $8.49 \mathrm{e}-05$ & -0.41 & $5.41 \mathrm{e}-09$ & 1.98 & $8.47 \mathrm{e}-05$ & -0.41 \\
\hline $1.0 \mathrm{e}-05$ & $2.91 \mathrm{e}-10$ & 1.25 & $9.16 \mathrm{e}-05$ & -0.03 & $3.24 \mathrm{e}-10$ & 1.22 & $7.25 \mathrm{e}-05$ & 0.06 \\
\hline
\end{tabular}

Table 2. Convergence of the difference quotients for the gradient and the Hessian of the reduced cost functional for Example 2 with $q=(6,6)^{T}$ and $\delta q=(1,1)^{T}$

\begin{tabular}{|c|c|c|c|c|c|c|c|c|}
\hline \multirow[b]{3}{*}{$\varepsilon$} & \multicolumn{4}{|c|}{ Discontinuous Galerkin } & \multicolumn{4}{|c|}{ Continuous Galerkin } \\
\hline & \multicolumn{2}{|c|}{ Gradient } & \multicolumn{2}{|c|}{ Hessian } & \multicolumn{2}{|c|}{ Gradient } & \multicolumn{2}{|c|}{ Hessian } \\
\hline & $e_{1}$ & Conv. & $e_{2}$ & Conv. & $e_{1}$ & Conv. & $e_{2}$ & Conv. \\
\hline $1.0 \mathrm{e}-00$ & $1.44 \mathrm{e}-01$ & - & $8.09 \mathrm{e}-02$ & - & $2.80 \mathrm{e}-01$ & - & $1.33 \mathrm{e}-01$ & - \\
\hline $1.0 \mathrm{e}-01$ & $1.36 \mathrm{e}-03$ & 2.02 & $7.76 \mathrm{e}-04$ & 2.01 & $2.59 \mathrm{e}-03$ & 2.03 & $1.27 \mathrm{e}-03$ & 2.02 \\
\hline $1.0 \mathrm{e}-02$ & $1.36 \mathrm{e}-05$ & 2.00 & $7.75 \mathrm{e}-06$ & 2.00 & $2.59 \mathrm{e}-05$ & 2.00 & $1.27 \mathrm{e}-05$ & 2.00 \\
\hline $1.0 \mathrm{e}-03$ & $1.36 \mathrm{e}-07$ & 1.99 & $4.32 \mathrm{e}-07$ & 1.25 & $2.59 \mathrm{e}-07$ & 1.99 & $3.97 \mathrm{e}-07$ & 1.50 \\
\hline $1.0 \mathrm{e}-04$ & $2.86 \mathrm{e}-09$ & 1.67 & $5.01 \mathrm{e}-05$ & -2.06 & $2.83 \mathrm{e}-09$ & 1.96 & $5.56 \mathrm{e}-06$ & -1.14 \\
\hline $1.0 \mathrm{e}-05$ & $5.94 \mathrm{e}-08$ & -1.31 & $2.18 \mathrm{e}-02$ & -2.63 & $9.95 \mathrm{e}-08$ & -1.54 & $2.00 \mathrm{e}-02$ & -3.55 \\
\hline
\end{tabular}

\subsection{Optimization}

In this subsection, we apply the two optimization algorithms described in Section 2 to the two considered optimization problems. For both examples, we present the results for the two time discretization schemes presented in Section 3. 
In Table 3 and Table 4, we show the progression of the norm of the gradient of the reduced functional $\left\|\nabla j_{h k}\right\|_{2}$ and the reduction of the cost functional $j_{h k}$ during the Newton iteration for Example 1 and Example 2, respectively.

The computations for Example 1 were done on a mesh consisting of 4096 hexahedral cells with diameter $h=0.0625$. The time interval $(0,1)$ is split into 100 slices of size $k=0.01$.

\begin{tabular}{|c|c|c|c|c|c|c|}
\hline \multirow[b]{2}{*}{ Step } & \multicolumn{3}{|c|}{ Discontinuous Galerkin } & \multicolumn{3}{|c|}{ Continuous Galerkin } \\
\hline & $n_{\mathrm{CG}}$ & $\left\|\nabla j_{h k}\right\|_{2}$ & $j_{h k}$ & $n_{\mathrm{CG}}$ & $\left\|\nabla j_{h k}\right\|_{2}$ & $j_{h k}$ \\
\hline 0 & - & $1.21 \mathrm{e}-01$ & $2.76 \mathrm{e}-01$ & - & $1.21 \mathrm{e}-01$ & $2.76 \mathrm{e}-01$ \\
\hline 1 & 2 & $4.99 \mathrm{e}-02$ & $1.34 \mathrm{e}-01$ & 2 & $4.98 \mathrm{e}-02$ & $1.34 \mathrm{e}-01$ \\
\hline 2 & 2 & $2.00 \mathrm{e}-02$ & $6.28 \mathrm{e}-02$ & 2 & $1.99 \mathrm{e}-02$ & $6.33 \mathrm{e}-02$ \\
\hline 3 & 3 & $7.61 \mathrm{e}-03$ & $2.94 \mathrm{e}-02$ & 3 & $7.62 \mathrm{e}-03$ & $3.00 \mathrm{e}-02$ \\
\hline 4 & 3 & $2.55 \mathrm{e}-03$ & $1.64 \mathrm{e}-02$ & 3 & $2.57 \mathrm{e}-03$ & $1.70 \mathrm{e}-02$ \\
\hline 5 & 3 & $6.03 \mathrm{e}-04$ & $1.32 \mathrm{e}-02$ & 3 & $6.21 \mathrm{e}-04$ & $1.37 \mathrm{e}-02$ \\
\hline 6 & 3 & $5.72 \mathrm{e}-05$ & $1.29 \mathrm{e}-02$ & 3 & $6.18 \mathrm{e}-05$ & $1.34 \mathrm{e}-02$ \\
\hline 7 & 3 & $6.37 \mathrm{e}-07$ & $1.29 \mathrm{e}-02$ & 3 & $7.62 \mathrm{e}-07$ & $1.34 \mathrm{e}-02$ \\
\hline 8 & 3 & $1.75 \mathrm{e}-10$ & $1.29 \mathrm{e}-02$ & 3 & $1.21 \mathrm{e}-10$ & $1.34 \mathrm{e}-02$ \\
\hline
\end{tabular}

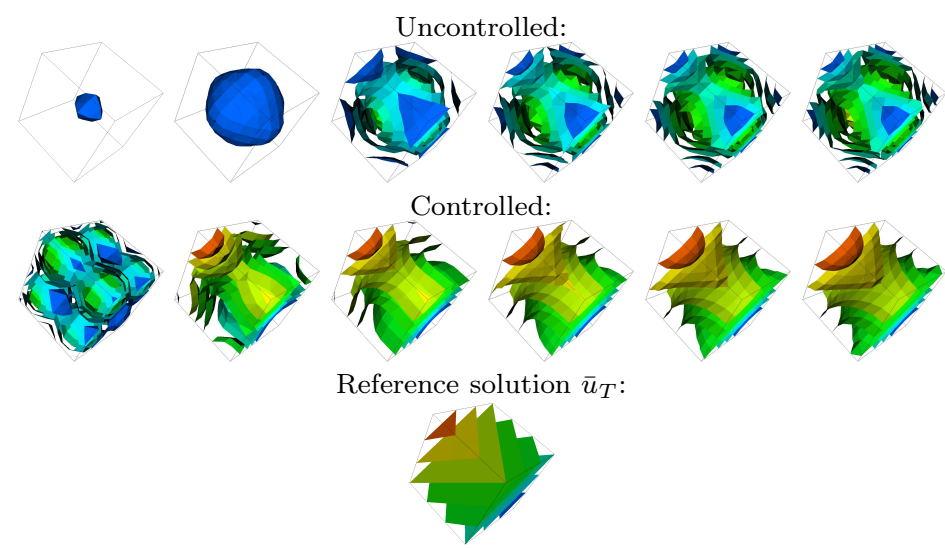

Figure 1. Solution of example problem 1 for time $t=0.0,0.2,0.4,0.6,0.8,1.0$ before and after optimization

For Example 2, we chose a quadrilateral mesh with mesh size $h=0.03125$ consisting of 1024 cells. The size of the time steps was set as $k=0.005$ corresponding to 200 time steps. In Table 4, we additionaly show the value of the estimated parameters during the optimization run. The values of "measurements" are taken from a solution of the state equation on a fine mesh consisting of 65536 cells with 5000 time steps for the "exact" values of parameters chosen as $q_{\text {exact }}=(7,9)^{T}$. 
Table 4. Results of the optimization loop with $\mathrm{dG}$ and $\mathrm{cG}$ discretization for Example 2

\begin{tabular}{|c|c|c|c|c|c|c|c|c|}
\hline \multirow[b]{2}{*}{ Step } & \multicolumn{4}{|c|}{ Discontinuous Galerkin } & \multicolumn{4}{|c|}{ Continuous Galerkin } \\
\hline & $n_{\mathrm{CG}}$ & $\left\|\nabla j_{h k}\right\|_{2}$ & $j_{h k}$ & $q$ & $n_{\mathrm{CG}}$ & $\left\|\nabla j_{h k}\right\|_{2}$ & $j_{h k}$ & $q$ \\
\hline 0 & - & $1.54 \mathrm{e}-02$ & $1.73 \mathrm{e}-03$ & $(6.00,6.00)^{T}$ & - & $1.25 \mathrm{e}-02$ & $1.23 \mathrm{e}-03$ & $(6.00,6.00)^{T}$ \\
\hline 1 & 2 & $5.37 \mathrm{e}-04$ & $4.53 \mathrm{e}-04$ & $(5.97,7.72)^{T}$ & 2 & $4.35 \mathrm{e}-04$ & $3.07 \mathrm{e}-03$ & $(6.06,7.43)^{T}$ \\
\hline 2 & 2 & $1.65 \mathrm{e}-04$ & $7.85 \mathrm{e}-05$ & $(6.80,8.52)^{T}$ & 2 & $1.29 \mathrm{e}-04$ & $4.75 \mathrm{e}-05$ & $(6.48,8.37)^{T}$ \\
\hline 3 & 2 & $3.44 \mathrm{e}-05$ & $5.56 \mathrm{e}-06$ & $(7.18,9.19)^{T}$ & 2 & $2.48 \mathrm{e}-05$ & $2.35 \mathrm{e}-06$ & $(6.87,8.84)^{T}$ \\
\hline 4 & 2 & $2.54 \mathrm{e}-06$ & $9.20 \mathrm{e}-07$ & $(7.35,9.39)^{T}$ & 2 & $1.47 \mathrm{e}-06$ & $9.29 \mathrm{e}-09$ & $(6.99,8.98)^{T}$ \\
\hline 5 & 2 & $1.66 \mathrm{e}-08$ & $8.91 \mathrm{e}-07$ & $(7.36,9.41)^{T}$ & 2 & $6.10 \mathrm{e}-09$ & $2.04 \mathrm{e}-10$ & $(6.99,8.99)^{T}$ \\
\hline 6 & 2 & $7.35 \mathrm{e}-13$ & $8.91 \mathrm{e}-07$ & $(7.36,9.41)^{T}$ & 1 & $5.89 \mathrm{e}-11$ & $2.04 \mathrm{e}-10$ & $(6.99,8.99)^{T}$ \\
\hline
\end{tabular}

We note that due to condition (21), for Example 1 the variant of the optimization algorithm, which only uses matrix-vector products of the Hessian is the more efficient one, whereas for Example 2 one should use the variant which builds up the entire Hessian.

\subsection{Windowing}

This subsection is devoted to the practical verification of the presented MultiLevel Windowing. For this, we consider Example 1 with dG time discretization on a grid consisting of 32768 cells performing 500 time steps. Table 5 demonstrates the reduction of the storage requirement described in Section 4. We can achieve a storage reduction about the factor 30 for both variants of the optimization loop. Thereby total number of steps only growths about the factor 3.2 for the algorithm with, and 4.0 for the algorithm without building up the entire Hessian.

Table 5. Reduction of the storage requirement due to Windowing in Example 1 with dG discretization and 32768 cells in each time step

\begin{tabular}{cccccc}
\hline & \multicolumn{2}{c}{ With Hessian } & & \multicolumn{2}{c}{ Without Hessian } \\
\cline { 2 - 3 } Factorization & Memory in MB & Time Steps & & Memory in MB & Time Steps \\
\hline 500 & 1236 & 45000 & & 274 & 35000 \\
\hline $5 \cdot 100$ & 259 & 80640 & & 58 & 87948 \\
$10 \cdot 50$ & 148 & 84690 & & 32 & 90783 \\
$2 \cdot 2 \cdot 5 \cdot 25$ & 78 & 120582 & & 17 & 118503 \\
$5 \cdot 10 \cdot 10$ & 59 & 114174 & & 13 & 113463 \\
$4 \cdot 5 \cdot 5 \cdot 5$ & 41 & 136512 & & 9 & 130788 \\
$2 \cdot 2 \cdot 5 \cdot 5 \cdot 5$ & 39 & 146646 & & 9 & 138663 \\
\hline
\end{tabular}

We remark that although the factorization $2 \cdot 2 \cdot 5 \cdot 25$ consists of more factors than the factorization $5 \cdot 10 \cdot 10$, both, the storage requirement and the total number of time steps are greater for first factorization than for the second one. The reason for this is the imbalance of the size of the different factors in $2 \cdot 2 \cdot 5 \cdot 25$. As showed in Section 4 , in the optimal factorization are all factors 
the same. So, it is evident, that a factorization as $5 \cdot 10 \cdot 10$ is more efficient than one where the size factors varies very much.

Table 5 also proves the asserted dependence of the condition when to use which variant of the optimization loop on the considered factorization on $M$. For the factorizations $5 \cdot 100$ and $10 \cdot 50$ the variant with building up the Hessian needs less forward steps than the other variant without building up the Hessian. However, for the remaining factorizations the situation is the opposite way around.

\section{References}

[1] Becker, R., 2001. Adaptive Finite Elements for Optimal Control Problems. Habilitationsschrift, Institut für Angewandte Mathematik, Universität Heidelberg.

[2] Becker, R., Braack, M., Meidner, D., Richter, T., Schmich, M., and Vexler, B., 2005. The finite element toolkit Gascoinge. URL http://www.gascoigne.uni-hd.de.

[3] Becker, R., Dunne, T., and Meidner, D., 2005. VisuSimple: An interactive VTK-based visualization and graphics/mpeg-generation program. URL http://www.visusimple.uni-hd.de.

[4] Becker, R., Meidner, D., and Vexler, B., 2005. RoDoBo: A C ++ library for optimization with stationary and nonstationary PDEs based on GascoIGne [2]. URL http://www.rodobo.uni-hd.de.

[5] Berggren, M., Glowinski, R., and Lions, J.-L., 1996. A computational approach to controllability issues for flow-related models. (I): Pointwise control of the viscous burgers equation. Int. J. Comput. Fluid Dyn., 7(3), 237-253.

[6] Bergounioux, M., Ito, K., and Kunisch, K., 1999. Primal-dual strategy for constrained optimal control problems. SIAM J. Control Optim., 37(4), 1176-1194.

[7] Ciarlet, P. G., 2002. The Finite Element Method for Elliptic Problems, volume 40 of Classics Appl. Math. SIAM, Philadelphia.

[8] Dautray, R. and Lions, J.-L., 1992. Mathematical Analysis and Numerical Methods for Science and Technology: Evolution Problems I, volume 5. Springer-Verlag, Berlin.

[9] Eriksson, K., Johnson, C., and Thomée, V., 1985. Time discretization of parabolic problems by the discontinuous Galerkin method. RAIRO Modelisation Math. Anal. Numer., 19, 611-643.

[10] Estep, D. and Larsson, S., 1993. The discontinuous Galerkin method for semilinear parabolic problems. RAIRO Modelisation Math. Anal. Numer., $\mathbf{2 7}(1), 35-54$. 
[11] Fursikov, A. V., 1999. Optimal Control of Distributed Systems: Theory and Applications, volume 187 of Transl. Math. Monogr. AMS, Providence.

[12] Griewank, A., 1992. Achieving logarithmic growth of temporal and spatial complexity in reverse automatic differentiation. Optim. Methods Softw., 1(1), 35-54.

[13] Griewank, A., 2000. Evaluating Derivatives, Principles and Techniques of Algorithmic Differentiation, volume 19 of Frontiers Appl. Math. SIAM, Philadelphia.

[14] Griewank, A. and Walther, A., 2000. Revolve: An implementation of checkpointing for the reverse or adjoint mode of computational differentiation. ACM Trans. Math. Software, 26(1), 19-45.

[15] Hinze, M. and Kunisch, K., 2001. Second order methods for optimal control of time-dependent fluid flow. SIAM J. Control Optim., 40(3), 925-946.

[16] Kunisch, K. and Rösch, A., 2002. Primal-dual active set strategy for a general class of constrained optimal control problems. SIAM J. Optim., 13(2), 321-334.

[17] Lions, J.-L., 1971. Optimal Control of Systems Governed by Partial Differential Equations, volume 170 of Grundlehren Math. Wiss. Springer-Verlag, Berlin.

[18] Litvinov, W. G., 2000. Optimization in Elliptic Problems With Applications to Mechanics of Deformable Bodies and Fluid Mechanics, volume 119 of Oper. Theory Adv. Appl. Birkhäuser Verlag, Basel.

[19] Rannacher, R. and Vexler, B., 2004. A priori error estimates for the finite element discretization of elliptic parameter identification problems with pointwise measurements. SIAM J. Control Optim. To appear.

[20] Tröltzsch, F., 1999. On the Lagrange-Newton-SQP method for the optimal control of semilinear parabolic equations. SIAM J. Control Optim., 38(1), 294-312. 